\title{
Observers with constant proper acceleration, constant proper jerk, and beyond
}

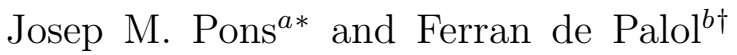 \\ a Departament de Física Quàntica i Astrofísica and Institut de Ciències del Cosmos (ICCUB), \\ Facultat de Física, Universitat de Barcelona, \\ Martí Franquès 1, 08028 Barcelona, Catalonia, Spain. and \\ ${ }^{b}$ Facultat de Física, Universitat de Barcelona, \\ Martí Franquès 1, 08028 Barcelona, Catalonia, Spain.
}

We discuss in Minkowski spacetime the differences between the concepts of constant proper $n$-acceleration and of vanishing $(n+1)$-acceleration. By $n$-acceleration we essentially mean the higher order time derivatives of the position vector of the trajectory of a point particle, adapted to Minkowski spacetime or eventually to curved spacetime. The 2-acceleration is known as the Jerk, the 3 -acceleration as the Snap, etc. As for the concept of proper $n$-acceleration we give a specific definition involving the instantaneous comoving frame of the observer and we discuss, in such framework, the difficulties in finding a characterization of this notion as a Lorentz invariant statement. We show how the Frenet-Serret formalism helps to address the problem. In particular we find that our definition of an observer with constant proper acceleration corresponds to the vanishing of the third curvature invariant $\kappa_{3}$ (thus the motion is three dimensional in Minkowski spacetime) together with the constancy of the first and second curvature invariants and the restriction $\kappa_{2}<\kappa_{1}$, the particular case $\kappa_{2}=0$ being the one commonly referred to in the literature. We generalize these concepts to curved spacetime, in which the notion of trajectory in a plane is replaced by the vanishing of the second curvature invariant $\kappa_{2}$. Under this condition, the concept of constant proper $n$-acceleration coincides with that of the vanising of the $(n+1)$-acceleration and is characterized by the fact that the first curvature invariant $\kappa_{1}$ is a $(n-1)$-degree polynomial of proper time. We illustrate some of our results with examples in Minkowski, de Sitter and Schwarzschild spacetimes.

*Electronic address: pons@fqa.ub.edu

$\dagger$ Electronic address: fdepalco@gmail.com 


\section{INTRODUCTION}

Although the physics in Minkowski spacetime as described by an inertial observer is indeed a very relevant description (one can just take a look at quantum field theory textbooks), the use of non inertial frames of reference, like the ones associated with accelerated observers, has become a very rich territory for our present understanding of new physical phenomena emerging from the quantum world. This is the case for instance of the thermal bath of Unruh radiation [1] that a constantly accelerated observer ${ }^{1}$ should experience, though it has not been experimentally verified yet [2] [3]. In addition, and just by taking into account the equivalence principle, one can relate the physics experienced by these accelerated observers in Minkowski spacetime with that of an observer hovering above the horizon of a Schwarzschild black hole. In fact the local description of a Schwarzschild black hole near the horizon is that of a Rindler spacetime, which is nothing but a patch of Minkowski spacetime described with the coordinates which are natural to the constantly accelerated observer (the coordinates in which this observer remains at rest).

Beyond the concept of acceleration there is an infinite tower of kinematical concepts with higher order time derivatives, which go under the names of Jerk, Snap, Crackle, etc. ${ }^{2}$. In Galilean mechanics these objects are trivial generalizations of the concept of acceleration, and their extension to special relativity has been introduced in [5] in a way that can be immediately generalized to curved spacetimes.

The main purpose of this paper is to elucidate the differences between the concepts of constant proper $n$-acceleration and vanishing $(n+1)$-acceleration. It is crucial in this respect to properly define the instantaneous comoving frame. We choose to do it in the simplest way available by implementing the map form the original frame to the instantaneous comoving frame as a pure boost, wih no additional rotations attached.

The organization of the paper is as follows. In section II we derive in a non conventional way the equations for the trajectories of the observer with constant proper acceleration in $4 \mathrm{~d}$ (or any dimension) Minkowski spacetime. From our analysis we notice that the trajectory of the observer with constant proper acceleration can be three dimensional (3d) or twodimensional $(2 \mathrm{~d})$. The $3 \mathrm{~d}$ case, which is new, is solved in section II C, The notion of Jerk is introduced in section IID and it is shown that its vanishing characterizes the motion of the observer with constant proper acceleration in the $2 \mathrm{~d}$ case, that is, when this motion takes place in a plane.

\footnotetext{
${ }^{1}$ From now on, by constantly accelerated observer we mean the observer with constant proper acceleration. Proper in the sense of being described in the instantaneous frame comoving with the observer.

${ }^{2}$ We learn in [4] that "This terminology goes back to a 1932 advertisement of Kellogg's Rice Crispies which 'merrily Snap, crackle, and pop in a bowl of milk' ". Here our use of these concepts is unrelated to the standard use in cosmology as higher order time derivatives of the scale factor in the Friedmann-LemaitreRobertson-Walker (FLRW) metric
} 
In the next section III we introduce all the tower of kinematical concepts with higher order time derivatives and in section [V] we address in detail the case of vanishing Snap and show that, unlike what would have been analogous to the vanishing Jerk case, it does not imply that the motion has constant proper Jerk, nor that the motion is restricted to a plane. We further deal with this issue in section $\mathrm{V}$ in which we show the difficulties of finding a Lorentz invariant statement for notions like that of a constant proper acceleration or a constant proper Jerk, etc. The Frenet-Serret formalism is introduced in section VI as a possible way out of these difficulties. In this way we are able to characterize the $3 \mathrm{~d}$ motion of the observer with constant proper acceleration (section VIA). We obtain in section VII solutions for the trajectory of an observer with vanishing $n$-acceleration and constant $(n-1)$ acceleration in a $2 \mathrm{~d}$ Minkowski spacetime. Conclusions and discussion are given in section VIII.

We finish with three appendices. The first is devoted to the circular motion in Minkowski spacetime; the second to constant proper acceleration in de Sitter spacetime; and the third to circular orbits in Schwarzschild spcetime.

\section{THE ACCELERATED OBSERVER IN 4D MINKOWSKI SPACETIME}

Given the trajectory $x^{\mu}(t(s))$ with $t$ the coordinate time and $s$ the proper time, its velocity with respect to the proper time is

$$
V(s)=\gamma\left(\begin{array}{c}
1 \\
x^{\prime}(t(s)) \\
y^{\prime}(t(s)) \\
z^{\prime}(t(s))
\end{array}\right)=\gamma\left(\begin{array}{c}
1 \\
\vec{r}^{\prime}(t(s))
\end{array}\right)
$$

where $x^{\prime}(t)$ is the derivative of $x(t)$ with respect to the coordinate time so that $\frac{d x^{i}}{d s}=\gamma x^{\prime}(t)$ with $\gamma=\frac{d t}{d s}=\frac{1}{\sqrt{1-\left(r^{\prime}\right)^{2}}}$ (we use dot, , , for proper time derivative $\frac{d}{d s}$ and prime, ', for coordinate time derivative $\left.\frac{d}{d t}\right)$. The definition of proper time guarantees $(V)^{2}=-1$.

We can prepare a family of Lorentz transformations $B(s)$ so that at any proper time $s$ we send the traveler to rest,

$$
V(s) \rightarrow B(s) V(s)=\left(\begin{array}{l}
1 \\
0 \\
0 \\
0
\end{array}\right)=: V_{p}, \forall s .
$$

There is an arbitrariness in choosing this family, because any boost sending the observer to rest can be followed by an arbitrary rotation without affecting the status of the observer 
as being at rest. Our choice will be the most simple available: that of a pure boost. Some of the results and definitions in this paper depend upon this choice. Other choices can be explored, for instance adding a rotation depending on the acceleration, but we think that ours is the first option to be considered. The pure boost that does the job is (with $t=t(s)$ ),

$$
B(s)=\left(\begin{array}{cccc}
\gamma & -\gamma x^{\prime}(t) & -\gamma y^{\prime}(t) & -\gamma z^{\prime}(t) \\
-\gamma x^{\prime}(t) & 1+\frac{\gamma^{2}}{\gamma+1} x^{\prime}(t)^{2} & \frac{\gamma^{2}}{\gamma+1} x^{\prime}(t) y^{\prime}(t) & \frac{\gamma^{2}}{\gamma+1} x^{\prime}(t) z^{\prime}(t) \\
-\gamma y^{\prime}(t) & \frac{\gamma^{2}}{\gamma+1} x^{\prime}(t) y^{\prime}(t) & 1+\frac{\gamma^{2}}{\gamma+1} y^{\prime}(t)^{2} & \frac{\gamma^{2}}{\gamma+1} y^{\prime}(t) z^{\prime}(t) \\
-\gamma z^{\prime}(t) & \frac{\gamma^{2}}{\gamma+1} x^{\prime}(t) z^{\prime}(t) & \frac{\gamma^{2}}{\gamma+1} y^{\prime}(t) z^{\prime}(t) & 1+\frac{\gamma^{2}}{\gamma+1} z^{\prime}(t)^{2}
\end{array}\right)
$$

The existence of non vanishing proper acceleration will be detected if, to do the same job, the boost necessary at proper time $s+\delta s$ is different from $B(s)$. In such case we will need an additional infinitesimal boost $\bar{B}(\delta s)$ so that $\bar{B}(\delta s) B(s)=B(s+\delta s)$. Thus, expanding to first order, $\bar{B}(\delta s)=(B(s)+\dot{B}(s) \delta s) B^{-1}(s)=I+\dot{B}(s) B^{-1}(s) \delta s$. The acceleration caused by $\bar{B}(\delta s)$ is, by definition,

$$
\frac{1}{\delta s}\left(\bar{B}(\delta s) V_{p}-V_{p}\right)=\dot{B}(s) B^{-1}(s) V_{p} .
$$

Thus the proper acceleration $A_{p}(s)$ (only spatial components) experienced by the observer at proper time $s$ is given by the action of $-\dot{B}(s) B^{-1}(s)$ on the rest velocity $V_{p}$ (a minus sign because the infinitesimal boost compensates for the acceleration at proper time $s)$. Using $\frac{d}{d s}=\gamma \frac{d}{d t}$ we get,

$$
A_{p}(s)=-\dot{B}(s) B^{-1}(s) V_{p}=\left(\begin{array}{c}
0 \\
a_{x} \\
a_{y} \\
a_{z}
\end{array}\right)=\left(\begin{array}{c}
0 \\
\vec{a}
\end{array}\right)
$$

with

$$
\vec{a}=\gamma^{2} \vec{r}^{\prime \prime}+\frac{\gamma}{\gamma+1} \gamma^{\prime} \vec{r}^{\prime}, \quad \gamma^{\prime}=\frac{d \gamma}{d t}=\frac{1}{2} \gamma^{3} \frac{d}{d t}\left(\vec{r}^{\prime}\right)^{2}
$$

Given the proper acceleration $A_{p}(s)$ in the instantaneous comoving frame, we can move back to the original coordinate system by applying the inverse boost $B^{-1}(s)$. Thus, taking into account that $V_{p}$ is constant, we obtain

$$
\begin{aligned}
A(s) & =B^{-1}(s) A_{p}(s)=-B^{-1}(s) \dot{B}(s) B^{-1}(s) V_{p}=\left(\frac{d}{d s} B^{-1}(s)\right) V_{p} \\
& =\frac{d}{d s}\left(B^{-1}(s) V_{p}\right)=\frac{d}{d s} V(s),
\end{aligned}
$$

that is, $A(s)=\dot{V}(s)$, as it could have been expected. Again, using $\frac{d}{d s}=\gamma \frac{d}{d t}$, one can also write

$$
A(s)=\left(\begin{array}{c}
0 \\
\gamma^{2} \vec{r}^{\prime \prime}
\end{array}\right)+\gamma^{\prime} V(s)=\left(\begin{array}{c}
\gamma \gamma^{\prime} \\
\vec{a}+\frac{\gamma^{2}}{\gamma+1} \gamma^{\prime} \vec{r}^{\prime}
\end{array}\right)
$$


and (note that $\gamma^{\prime}=\vec{a} \cdot \vec{r}^{\prime}$ )

$$
A^{2}=A_{p}^{2}=\vec{a}^{2}=\gamma^{4}\left(\vec{r}^{\prime \prime}\right)^{2}+\left(\gamma^{\prime}\right)^{2}
$$

\section{A. Constant proper acceleration}

Let us examine the case of constant proper acceleration, $\vec{a}=\vec{a}_{0}$ constant. In our view this constancy will correspond to Einstein's intuitive definition of uniform acceleration [6].

The equation of motion in the original coordinate system is, from (II.3),

$$
\vec{r}^{\prime \prime}=\frac{1}{\gamma^{2}} \vec{a}_{0}-\frac{1}{\gamma(\gamma+1)} \gamma^{\prime} \vec{r}^{\prime}
$$

This equation is more easily managed with the use of proper time $s$. Equation (II.6) then becomes (note that $\dot{t}=\gamma$ )

$$
\ddot{\vec{r}}(s)=\vec{a}_{0}+\frac{\ddot{t}(s)}{\dot{t}(s)+1} \dot{\vec{r}}(s) .
$$

A parametrization of the velocity which guarantees that $\dot{t}(s)>0, \forall s$, is the following,

$$
V(s)=\left(\begin{array}{c}
\dot{t}(s) \\
\dot{\vec{r}}(s)
\end{array}\right)=\left(\begin{array}{c}
\cosh (f(s)) \\
\sinh (f(s)) \vec{n}(s)
\end{array}\right)
$$

with $\vec{n}(s)$ a unitary euclidean 3-vector. We obtain, from (II.7)

$$
\dot{\vec{n}}=\frac{1}{\sinh (f)}\left(\vec{a}_{0}-\dot{f} \vec{n}\right) .
$$

An important consequence of this equation (II.9) is that it shows that the motion of $\vec{n}(s)$ in 3 -space is at most in a plane containing $\vec{a}_{0}$. From $(\dot{\vec{n}}, \vec{n})=0$ we infer that $\dot{f}=\left(\vec{a}_{0}, \vec{n}\right)$. Inserting II.9 into its derivative $\ddot{f}=\left(\vec{a}_{0}, \dot{\vec{n}}\right)$ we obtain the equation for $f$,

$$
\ddot{f} \sinh (f)=a_{0}^{2}-(\dot{f})^{2} .
$$

An obvious solution is $f(s)=a_{0}\left(s-s_{0}\right)$ with arbitrary $s_{0}$ and $a_{0}=\left|\vec{a}_{0}\right|$. In this case $\dot{\vec{n}}=0$ and $\vec{n}=\frac{\vec{a}_{0}}{a_{0}}$ and the motion takes place in a plane (2d) in Minkowski spacetime. For any other solution, having seen from (II.9) that the motion of $\vec{n}(s)$ in 3-space is in a plane, we infer that the trajectory is $3 \mathrm{~d}$ in Minkowski spacetime.

\section{B. The 2d solution}

Let us focus now on this $2 \mathrm{~d}$ solution. The trajectory can be easily obtained by integrating (II.8) with constant $\vec{n}$ and $f(s)=a_{0}\left(s-s_{0}\right)$. One can also work directly with (II.6) and write $\vec{r}^{\prime}(t)=\lambda^{\prime}(t) \vec{a}_{0}$, for some function $\lambda^{\prime}(t)$, This equation integrates to $\vec{r}(t)=\vec{r}_{0}+\left(\lambda(t)-\lambda_{0}\right) \vec{a}_{0}$, 
with $\vec{r}_{0}=\vec{r}\left(t_{0}\right), \lambda_{0}=\lambda\left(t_{0}\right)$ for an initial time $t_{0}$. Substituting it in II.6) we get for $\lambda(t)$ the equation (with $a_{0}=\left|\vec{a}_{0}\right|$ )

$$
\frac{\lambda^{\prime \prime}}{\left(1-\left(\lambda^{\prime}\right)^{2} a_{0}^{2}\right)^{\frac{3}{2}}}=1
$$

with general solution

$$
a_{0}^{2}\left(\lambda-\lambda_{0}\right)^{2}-\left(t-t_{0}\right)^{2}=\frac{1}{a_{0}^{2}} \Rightarrow\left(\vec{r}-\vec{r}_{0}\right)^{2}-\left(t-t_{0}\right)^{2}=\frac{1}{a_{0}^{2}},
$$

which are the expected equations for the $2 \mathrm{~d}$ constantly accelerated oberver.

\section{The 3d solution}

To find the $3 \mathrm{~d}$ solution of the constantly accelerated observer it proves useful to adopt a parametrization different from that of (II.8). We only need to consider a 3d Minkowski spacetime, and we parametrize the velocity (with respect to proper time $s$ ) of the trajectory with

$$
V=\left(\begin{array}{c}
V_{0} \\
\vec{V}
\end{array}\right) \quad \text { with } \quad \vec{V}=\left(\begin{array}{l}
V_{1} \\
V_{2}
\end{array}\right)=v(s)\left(\begin{array}{c}
\sin (f(s)) \\
\cos (f(s))
\end{array}\right),
$$

with $v(s)$ and $f(s)$ to be determined by the conditions of constant proper acceleration. To guarantee that $(V)^{2}=-1$ notice that $V_{0}$ gets determined as $V_{0}=\sqrt{v^{2}+1}$.

The pure boost of eq. (II.1), now with a different notation, can be written as

$$
B=\left(\begin{array}{cc}
V_{0} & -\vec{V} \\
-\vec{V} & I+\frac{1}{V_{0}+1} \vec{V} \otimes \vec{V}
\end{array}\right)
$$

The action of $B(s)$ on the acceleration $A=\frac{d}{d s} V$ defines the proper acceleration $A_{p}$,

$$
B A=A_{p}=\left(\begin{array}{c}
0 \\
\overrightarrow{A_{p}}
\end{array}\right)
$$

and now $\vec{A}_{p}$ is required to be a constant vector $\vec{a}_{0}$. Using II.12, II.13, we find

$$
\overrightarrow{A_{p}}=\frac{\dot{v}}{V_{0}}\left(\begin{array}{c}
\sin (f(s)) \\
\cos (f(s))
\end{array}\right)+v \dot{f}\left(\begin{array}{c}
\cos (f(s)) \\
-\sin (f(s))
\end{array}\right)=\vec{a}_{0}=a_{0}\left(\begin{array}{c}
\cos \theta_{0} \\
\sin \theta_{0}
\end{array}\right)
$$

with constant $\theta_{0}$. From this expression we infer

$$
\frac{\dot{v}}{V_{0}}=a_{0} \sin \left(f(s)+\theta_{0}\right), \quad v \dot{f}=a_{0} \cos \left(f(s)+\theta_{0}\right),
$$

and, consequently,

$$
\frac{\dot{v}}{V_{0}} \cos \left(f(s)+\theta_{0}\right)=v \dot{f} \sin \left(f(s)+\theta_{0}\right)=-v \frac{d}{d s} \cos \left(f(s)+\theta_{0}\right),
$$


or, defining $g(s)=\cos \left(f(s)+\theta_{0}\right)$,

$$
\frac{\dot{g}}{g}=-\frac{\dot{v}}{v V_{0}}
$$

which integrates to $\left(V_{0}=\sqrt{v^{2}+1}\right)$

$$
g \frac{v}{1+V_{0}}=c
$$

with $c$ a constant that must be $|c|<1$ because $|g| \leq 1$ and $\left|\frac{v}{1+V_{0}}\right|<1$. Thus

$$
\cos \left(f(s)+\theta_{0}\right)=g(s)=\frac{c\left(1+V_{0}\right)}{v},
$$

which can be inserted into the first equation in II.14,

$$
\frac{\dot{v}}{V_{0}}=a_{0} \sqrt{1-g^{2}}=a_{0} \sqrt{1-\frac{c^{2}\left(1+V_{0}\right)^{2}}{v^{2}}},
$$

Thus,

$$
\dot{V}_{0}=\frac{v \dot{v}}{V_{0}}=a_{0} \sqrt{v^{2}-c^{2}\left(1+V_{0}\right)^{2}}=a_{0} \sqrt{\left(1+V_{0}\right)\left(d\left(1+V_{0}\right)-2\right)}
$$

with $d=1-c^{2}$ which implies $0<d \leq 1$. Finally, defining the new variable $z(s)$ from $1+V_{0}=\frac{1}{d}(z+1)$, the last equation becomes

$$
\dot{z}=\sqrt{d} a_{0} \sqrt{z^{2}-1} \Rightarrow z(s)=\cosh \left(\sqrt{d} a_{0}\left(s-s_{0}\right)\right) .
$$

With the choice $\theta_{0}=0$, which is always reachable by rotating the spatial coordinates, we obtain, using (II.15),

$$
\begin{aligned}
V_{1} & =v \sin (f(s))=v \sqrt{1-g^{2}}=\sqrt{\left(1+V_{0}\right)\left(d\left(1+V_{0}\right)-2\right)}=\frac{1}{\sqrt{d}} \sqrt{z^{2}-1} \\
& =\frac{1}{\sqrt{d}} \sinh \left(\sqrt{d} a_{0}\left(s-s_{0}\right)\right), \\
V_{2} & =v \cos (f(s))=v g=c\left(1+V_{0}\right)=\frac{\sqrt{1-d}}{d}(z+1) \\
& =\frac{\sqrt{1-d}}{d}\left(\cosh \left(\sqrt{d} a_{0}\left(s-s_{0}\right)\right)+1\right) .
\end{aligned}
$$

Thus the solution for $V$ is

$$
V=\left(\begin{array}{c}
V_{0} \\
\vec{V}
\end{array}\right)=\left(\begin{array}{c}
\frac{1}{d}\left(\cosh \left(\sqrt{d} a_{0}\left(s-s_{0}\right)\right)+1\right)-1 \\
\frac{1}{\sqrt{d}} \sinh \left(\sqrt{d} a_{0}\left(s-s_{0}\right)\right) \\
\frac{\sqrt{1-d}}{d}\left(\cosh \left(\sqrt{d} a_{0}\left(s-s_{0}\right)\right)+1\right)
\end{array}\right)
$$

and for the position vector $X$,

$$
X(s)=\left(\begin{array}{l}
t \\
x \\
y
\end{array}\right)=X\left(s_{0}\right)+\left(\begin{array}{c}
\frac{1}{a_{0} d^{3 / 2}} \sinh \left(a_{0} \sqrt{d}\left(s-s_{0}\right)\right)+\left(\frac{1-d}{d}\right)\left(s-s_{0}\right) \\
\frac{1}{a_{0} d}\left(\cosh \left(a_{0} \sqrt{d}\left(s-s_{0}\right)\right)-1\right) \\
\frac{\sqrt{1-d}}{a_{0} d^{3 / 2}}\left(a_{0} \sqrt{d}\left(s-s_{0}\right)+\sinh \left(a_{0} \sqrt{d}\left(s-s_{0}\right)\right)\right)
\end{array}\right)
$$


The motion in 2-space, coordinates $(x, y)$, exhibits asymptotes. Note that $d$ is related to the angle $\delta$ (the "scattering angle") in the $(x, y)$ plane between the ingoing $s \rightarrow-\infty$ and the outgoing $s \rightarrow \infty$ directions, $d=\left(\sin \frac{\delta}{2}\right)^{2}$. For $d=1(\delta=\pi)$ we recover the 2 d solution described in (II.11), with a turning point for the trajectory in 1-space.

$$
X_{(d=1)}(s)=\frac{1}{a_{0}}\left(\begin{array}{c}
\sinh \left(a_{0}\left(s-s_{0}\right)\right) \\
\cosh \left(a_{0}\left(s-s_{0}\right)\right)-1 \\
0
\end{array}\right) \text {, }
$$

We will revisit this solution in section VIA.

\section{Enters the Jerk}

As anticipated above, we will see in section $\mathrm{V}$ that the concept of constant proper acceleration, as defined by way of (II.2) with constant $\vec{a}$, depends on the coordinate frame used ${ }^{3}$, and this is not completely satisfactory. One should really say that a trajectory exhibits constant proper acceleration in Minkowski spacetime if there exist a coordinate frame for which the object $\vec{a}$ defined in (II.3) is constant. There is nothing wrong with such definition but it is much more convenient and elegant to have a definition in the form of an invariant statement -a covariant equation which in addition we wish to carry over to general relativity. In the case of the $2 \mathrm{~d}$ motion, this is achieved with the notion of the Jerk.

The Jerk vector $\Sigma$ (we follow the notation of [5]) is defined as the component orthogonal to the velocity of the proper time derivative of the acceleration (see section III for more details),

$$
\Sigma=\frac{d A}{d s}-(A)^{2} V
$$

Now it is easy to show that for an observer with constant proper acceleration and $2 \mathrm{~d}$ motion the Jerk vector vanishes -which is an invariant statement. In fact $\Sigma$ is orthogonal to $V$ by construction (see next section III for details) and if the proper acceleration is constant then $\Sigma$ is also orthogonal to $A$ because -considering (II.18) $-A$ is orthogonal to $V$ and has $(A)^{2} \equiv(A, A)$ constant. Then if the motion is in a plane and $\Sigma$ is orthogonal both to $V$ and $A$, it must vanish.

On the other hand, the vanishing of the Jerk leads to the constantly accelerated trajectories in $2 \mathrm{~d}$ motion examined above, as we now show. $\Sigma=0$ implies $\dot{A}=(A)^{2} V$, so we have $(A \cdot \dot{A})=0$, which means $(A)^{2}$ is constant, $(A)^{2}=a_{0}^{2}$ with $a_{0}=|A|$. Then we have (defining

\footnotetext{
${ }^{3}$ This issue only arises if the Minkowski spacetime has more than 2 dimensions.
} 
the normalized vector $\left.A_{N}:=\frac{A}{|A|}\right)$ the equations,

$$
\begin{gathered}
\dot{V}=A=: a_{0} A_{N} \\
\dot{A}_{N}=\frac{1}{a_{0}} \dot{A}=a_{0} V,
\end{gathered}
$$

which show that the solution is indeed $2 \mathrm{~d}$. The solution can be written as

$$
V(s)=\cosh \left(a_{0} s\right) V(0)+\sinh \left(a_{0} s\right) A_{N}(0)
$$

where the initial conditions are restricted to satisfy $V(0)^{2}=-1, A_{N}(0)^{2}=1$ and $\left(V(0) \cdot A_{N}(0)\right)=0$.

The trajectory is

$$
X(s)=X(0)+\frac{1}{a_{0}}\left(\sinh \left(a_{0} s\right) V(0)+\left(\cosh \left(a_{0} s\right)-1\right) A_{N}(0)\right),
$$

that is, the well known constantly accelerated observer.

If we parametrize the initial conditions in a $2 \mathrm{~d}$ subspace of Minkowski,

$$
V(0)=\left(\begin{array}{c}
\cosh \left(a_{0} s_{0}\right) \\
-\sinh \left(a_{0} s_{0}\right)
\end{array}\right) \quad A_{N}(0)=\left(\begin{array}{c}
-\sinh \left(a_{0} s_{0}\right) \\
\cosh \left(a_{0} s_{0}\right)
\end{array}\right),
$$

for some real $s_{0}$, we may write

$$
V(s)=\left(\begin{array}{c}
\cosh \left(a_{0}\left(s-s_{0}\right)\right) \\
\sinh \left(a_{0}\left(s-s_{0}\right)\right)
\end{array}\right),
$$

which is nothing but (II.8) with $f(s)=a_{0}\left(s-s_{0}\right)$.

We conclude that, in Minkowski spacetime -no matter the number of dimensions-, if the motion takes place in a plane $(2 \mathrm{~d})$, the observer with constant proper acceleration is identified by the vanishing of the Jerk, $\Sigma=0$.

\section{In Minkowski spacetime:}

Jerk $=0 \Leftrightarrow$ Constant proper acceleration + Two-dimensional motion.

\section{E. Extension to curved spacetime}

The important novelty is that this statement on the vanishing of the Jerk can be exported to curved spacetime. In fact, the definition of the Jerk (and all the tower of kinematical concepts) is extended to curved spacetime with the replacement of the partial derivative by the covariant derivative,

$$
\partial_{\mu} \rightarrow \nabla_{\mu} \quad \Rightarrow \quad \frac{d}{d s} A^{\mu} \rightarrow \frac{d}{d s} A^{\mu}+V^{\rho} \Gamma_{\rho \nu}^{\mu} A^{\nu},
$$


and requiring the connection to satisfy the metricity condition. The reason for this is the following. Let us define $\dot{A}$ in curved spacetime as the geometric object displayed just above, that is, $\dot{A}=\frac{d}{d s} A+V \Gamma A$, then, in order to guarantee that results like $(\dot{A}, V)=-(A)^{2}$, or $\frac{d}{d s}(A, A)=2(\dot{A}, A)$, still hold in curved spacetime, we need the covariant derivative of the metric to vanish. With this condition the Lorentz covariant expressions in this section and the next sections can be exported to curved spacetime. Since the Levi Civita connection is metric compatibile, we conclude that in the framework of general relativity the vanishing of the Jerk ensures that the observer undergoes constant proper acceleration. This is also true for other generally covariant theories with metric compatibile connections [7], like the Einstein-Cartan theory, in which torsion is sourced by fermionic matter, or teleparallel gravity, defined with the Weitzenböck connection.

In curved spacetime:

The vanishing of the Jerk implies that the observer has constant proper acceleration.

We give an example in Appendix B of constant proper acceleration in de Sitter spacetime.

\section{JERK, SNAP, CRACKLE...}

Acceleration, Jerk, Snap, Crackle, Pop, etc., are a family of kinematical concepts that are built with the second, third, fourth, etc, derivatives -with respect to proper time- of the trajectory (or with the first, second, third derivatives of the velocity vector). Each one is defined (see [5]) as the component orthogonal to the velocity of the proper time derivative of the previous object. This definition guarantees a threefold condition, namely, that all these objects are spacelike, that in the instantaneous comoving frame of the observer, they have only spatial components, as it already happens with the acceleration, and that if one of them vanishes, say the Jerk, all the higher derivative objects, say the Snap, Crackle, etc, will vanish as well.

Thus acceleration $A$, defined as (see (II.4))

$$
A=\frac{d}{d s} V
$$

is already orthogonal to the velocity, since $(V)^{2}=(V, V)=-1$. The Jerk $\Sigma$ is then defined as

$$
\Sigma=\left(\frac{d}{d s} A\right)_{\perp}=\frac{d}{d s} A-\frac{1}{(V)^{2}}\left(\frac{d}{d s} A, V\right) V=\frac{d}{d s} A+\left(\frac{d}{d s} A, V\right) V=\frac{d}{d s} A-(A)^{2} V,
$$

and the Snap $\Xi$,

$$
\Xi=\left(\frac{d}{d s} \Sigma\right)_{\perp}=\frac{d}{d s} \Sigma-\frac{1}{(V)^{2}}\left(\frac{d}{d s} \Sigma, V\right) V=\frac{d}{d s} \Sigma+\left(\frac{d}{d s} \Sigma, V\right) V=\frac{d}{d s} \Sigma-(\Sigma, A) V,
$$


and so on.

In order to generalize these expressions above, we adopt the notation of $A_{1}$ for the acceleration $A ; A_{2}$ for the "second acceleration" or Jerk, $A_{2}=\Sigma ; A_{3}$ for the "third acceleration" or Snap, $A_{3}=\Xi$, etc. In general the $n$-acceleration will be denoted by $A_{n}$. The recursive definition of these objects is then

$$
A_{1}=\frac{d}{d s} V, \quad A_{(n+1)}=\left(\frac{d}{d s} A_{n}\right)_{\perp}=\frac{d}{d s} A_{n}-\left(A_{n}, A_{1}\right) V
$$

Having the case of vanishing Jerk been discussed in the previous section, we shall address next the case of vanishing Snap.

\section{VANISHING SNAP}

Let us discuss the case $\Xi=0$. This implies $\frac{d}{d s} \Sigma=(\Sigma, A) V$ and therefore $\frac{d}{d s}\left(\Sigma^{2}\right)=$ $2(\Sigma, \dot{\Sigma})=0$, which means that $\Sigma^{2}$ is constant, $\Sigma^{2}=\sigma^{2}$ with $\sigma$ real -and we take $\sigma>0$ (the $\sigma=0$ case corresponds to the vanishing Jerk case discussed in the previous section). Note also that $\frac{d}{d s}(\Sigma, A)=\Sigma^{2}=\sigma^{2}$ and therefore $(\Sigma, A)=\sigma^{2}\left(s-s_{0}\right)$ with the arbitrary real constant $s_{0}$. Now we can compute $\frac{d}{d s}\left(A^{2}\right)=2(\Sigma, A)=2 \sigma^{2}\left(s-s_{0}\right)$ and we get $A^{2}=$ $\sigma^{2}\left(s-s_{0}\right)^{2}+\beta^{2}$, where we have implemented the condition of $A^{2} \geq 0$ with $\beta$ real. We end up with

$$
\begin{aligned}
A^{2} & =\sigma^{2}\left(s-s_{0}\right)^{2}+\beta^{2}, \\
(\Sigma, A) & =\sigma^{2}\left(s-s_{0}\right), \\
\Sigma^{2} & =\sigma^{2}, \quad \text { with } \sigma>0, \beta \geq 0 .
\end{aligned}
$$

Note from IV.1 that $\left(A-\left(s-s_{0}\right) \Sigma\right)^{2}=\beta^{2}$. Also, $\frac{d}{d s}\left(A-\left(s-s_{0}\right) \Sigma\right)=\dot{A}-\Sigma-\left(s-s_{0}\right) \dot{\Sigma}=$ $A^{2} V-\left(s-s_{0}\right)(\Sigma, A) V=\beta^{2} V$. It is relevant to distinguish the case $\beta=0$ from the rest.

\section{A. The case $\beta=0$}

From these last equations we infer that $\beta=0$ implies that $A-\left(s-s_{0}\right) \Sigma$ is a constant lightlike vector, so $A\left(s_{0}\right)$ would be a lightlike vector, which is incompatible with it being orthogonal to $V\left(s_{0}\right)$ unless $A-\left(s-s_{0}\right) \Sigma$ vanishes. We conclude that in this case $A=\left(s-s_{0}\right) \Sigma$. That is

$$
\begin{aligned}
& \dot{V}=\left(s-s_{0}\right) \Sigma \\
& \dot{\Sigma}=\sigma^{2}\left(s-s_{0}\right) V
\end{aligned}
$$

the second equation coming from the vanishing Snap condition and IV.1) . 
We see that this case corresponds to motion in a plane (i.e., 2d) in Minkowski spacetime. The solution is

$$
\begin{aligned}
& V(s)=\cosh \left(\frac{1}{2} \sigma\left(s-s_{0}\right)^{2}\right) V\left(s_{0}\right)+\sinh \left(\frac{1}{2} \sigma\left(s-s_{0}\right)^{2}\right) \frac{1}{\sigma} \Sigma\left(s_{0}\right), \\
& \Sigma(s)=\sigma \sinh \left(\frac{1}{2}\left(s-s_{0}\right)^{2}\right) V\left(s_{0}\right)+\cosh \left(\frac{1}{2} \sigma\left(s-s_{0}\right)^{2}\right) \Sigma\left(s_{0}\right)
\end{aligned}
$$

(Note that we may always keep $\sigma>0$ ) If we parametrize the initial conditions

$$
V\left(s_{0}\right)=\left(\begin{array}{c}
\cosh (\alpha) \\
\sinh (\alpha) \vec{n}_{0}
\end{array}\right) \quad \Sigma\left(s_{0}\right)=\sigma\left(\begin{array}{c}
\sinh (\alpha) \\
\cosh (\alpha) \vec{n}_{0}
\end{array}\right)
$$

for some real $\alpha$ and a unit norm constant 3 -vector $\vec{n}_{0}, \vec{n}_{0}^{2}=1$, then the general solution for $V$ is expressed with an arbitrary second degree polynomial with real coefficients $P_{2}(s)=$ $\frac{1}{2} \sigma\left(s-s_{0}\right)^{2}+\alpha$

$$
V(s)=\left(\begin{array}{c}
\cosh \left(P_{2}(s)\right) \\
\sinh \left(P_{2}(s)\right) \vec{n}_{0}
\end{array}\right) \quad \Sigma(s)=\sigma\left(\begin{array}{c}
\sinh \left(P_{2}(s)\right) \\
\cosh \left(P_{2}(s)\right) \vec{n}_{0}
\end{array}\right)
$$

with $P_{2}$ an arbitrary second degree polynomial with real coeffients and with $\vec{n}_{0}$ a normalized constant 3 -vector. In fact we can restrict $\sigma$ to be positive by eventually absorbing a minus sign within $\vec{n}_{0}$ in (IV.4. When we send the oberver to rest by using the appropriate boost, we get the constant proper Jerk,

$$
\Sigma_{p}(s)=\sigma\left(\begin{array}{c}
0 \\
\vec{n}_{0}
\end{array}\right)
$$

Note the change of the degree of the polynomial when compared with (II.20). Now the argument within the hyperbolic trigonometric functions is a second degree polynomial, instead of the first degree polynomial which we found for the the solution of the vanishing Jerk case, (II.20). This observation opens a natural way to explore $2 \mathrm{~d}$ solutions for the vanishing Crackle (constant proper Snap) case and higher derivative cases in general. This issue will be addressed in section VII.

\section{B. The case $\beta \neq 0$}

Collecting the expressions in the first part of this section, and defining the vector $D=$ $A-\left(s-s_{0}\right) \Sigma$, we have from the previous analysis the equations

$$
\begin{aligned}
& \dot{V}=A=D+\left(s-s_{0}\right) \Sigma \\
& \dot{D}=\beta^{2} V \\
& \dot{\Sigma}=\sigma^{2}\left(s-s_{0}\right) V
\end{aligned}
$$


with $(V)^{2}=-1,(D)^{2}=\beta^{2},(\Sigma)^{2}=\sigma^{2}$ and all three vectors orthogonal. So in this case the motion takes place in three dimensions. The equation for the 3 -vector $V$ is obtained from (IV.6) as

$$
\dddot{V}-\left(\sigma^{2}\left(s-s_{0}\right)^{2}+\beta^{2}\right) \dot{V}-3 \sigma^{2}\left(s-s_{0}\right) V=0
$$

With the proper time rescaled to $\tau=\sqrt{\sigma}\left(s-s_{0}\right)$, and defining $W=\frac{1}{\beta} D, Z=\frac{1}{\sigma} \Sigma$, the system IV.6f becomes simplified (with $V^{\prime}=\frac{d}{d \tau} V$, etc.),

$$
\begin{aligned}
V^{\prime} & =c W+\tau Z \\
W^{\prime} & =c V \\
Z^{\prime} & =\tau V,
\end{aligned}
$$

with $c=\frac{\beta}{\sqrt{\sigma}}$ and the orthonormal system $V, W, Z$, with $(V)^{2}=-1,(W)^{2}=1,(Z)^{2}=1$.

In this equation, $V, W, Z$ are three orthonormal vectors - $V$ timelike, $W$ and $Z$ spacelike- in an $n$-Minkoswki spacetime. In fact, since their derivatives in (IV.8) are linear combinations of the three vectors themselves, we infer that the motion is at most 3-dimensional, and so we continue with a 3d Minkoswki spacetime. A solution for $V(\tau)$ will be of the type $V(\tau)=$ $V_{0}(\tau) V(0)+V_{1}(\tau) W(0)+V_{2}(\tau) Z(0)$ and since $V(0), W(0), Z(0)$, span an orthonormal basis, we can take

$$
V(0)=\left(\begin{array}{l}
1 \\
0 \\
0
\end{array}\right), \quad W(0)=\left(\begin{array}{l}
0 \\
1 \\
0
\end{array}\right), \quad Z(0)=\left(\begin{array}{l}
0 \\
0 \\
1
\end{array}\right)
$$

Analogously to (IV.7), a single equation for $V$ is obtained from (IV.8) as

$$
V^{\prime \prime \prime}-\left(\tau^{2}+c^{2}\right) V^{\prime}-3 \tau V=0
$$

We have not found analytic solutions for (IV.8) nor for its consequence (IV.9), but we can examine nevertheless the behaviour of the solutions in the asymptotic time regime $|\tau|>>c$ and in the "mid-term" regime $|\tau|<<c$.

For large $|\tau|($ IV.8) is approximated by

$$
\begin{aligned}
& V^{\prime}=\tau Z \\
& Z^{\prime}=\tau V,
\end{aligned}
$$

which is just the system considered in (IV.2), that is, the $2 \mathrm{~d}$ motion (one spatial dimension) with constant proper Jerk. In sharp contrast, for $|\tau|<<c$ the system becomes close to

$$
\begin{gathered}
V^{\prime}=c W \\
W^{\prime}=c V,
\end{gathered}
$$


which is nothing but the $2 \mathrm{~d}$ motion (one spatial dimension) with constant proper acceleration (vanishing Jerk), already discussed in (II.19). Therefore (IV.8) -and (IV.6)- describes a motion which is composite of these two fundamental motions, now in $3 \mathrm{~d}$ (two spatial dimensions).

\section{Vanishing Snap vs constant proper Jerk}

We have seen in section IID that vanishing Jerk implies motion in a plane (2d in Minkowski) and constant proper acceleration. On the other hand, we have seen in the previous section IVB that vanishing Snap does not imply in general $2 \mathrm{~d}$ motion. We may wonder now whether a vanishing Snap condition will at least imply a constant proper Jerk. The answer is in the negative. Let us examine it.

To send the observer to the rest frame we will apply the pure boost (II.1), equivalently written as

$$
B=\left(\begin{array}{cccc}
V_{0} & -V_{1} & -V_{2} & -V_{3} \\
-V_{1} & 1+\frac{1}{V_{0}+1} V_{1}^{2} & \frac{1}{V_{0}+1} V_{1} V_{2} & \frac{1}{V_{0}+1} V_{1} V_{3} \\
-V_{2} & \frac{1}{V_{0}+1} V_{1} V_{2} & 1+\frac{1}{V_{0}+1} V_{2}^{2} & \frac{1}{V_{0}+1} V_{2} V_{3} \\
-V_{3} & \frac{1}{V_{0}+1} V_{1} V_{3} & \frac{1}{V_{0}+1} V_{2} V_{3} & 1+\frac{1}{V_{0}+1} V_{3}^{2}
\end{array}\right) \equiv\left(\begin{array}{cc}
V_{0} & -\vec{V} \\
-\vec{V} & I+\frac{1}{V_{0}+1} \vec{V} \otimes \vec{V}
\end{array}\right)
$$

Let us add to the vanishing Snap condition that of the constant proper Jerk. By this we mean that when we send $\Sigma$ to the comoving frame, the resulting object, $\Sigma_{p}$, is a constant vector,

$$
B \Sigma=\left(\begin{array}{c}
0 \\
\vec{b}_{0}
\end{array}\right)
$$

with $\vec{b}_{0}$ a constant 3 -vector. We obtain from IV.13 and IV.12, with $\Sigma=\left(\Sigma_{0}, \vec{\Sigma}\right)$,

$$
\vec{\Sigma}-\frac{\Sigma_{0}}{V_{0}+1} \vec{V}=\vec{b}_{0}
$$

which reads, in 4-vector language,

$$
\Sigma-\frac{\Sigma_{0}}{V_{0}+1} V=\left(\begin{array}{c}
\frac{\Sigma_{0}}{V_{0}+1} \\
\overrightarrow{b_{0}}
\end{array}\right)
$$

which implies, by derivating with respect to the proper time,

$$
\dot{\Sigma}-\frac{d}{d s}\left(\frac{\Sigma_{0}}{V_{0}+1}\right) V-\frac{\Sigma_{0}}{V_{0}+1} \dot{V}=\left(\begin{array}{c}
\frac{d}{d s}\left(\frac{\Sigma_{0}}{V_{0}+1}\right) \\
\overrightarrow{0}
\end{array}\right)
$$

and using (IV.6),

$$
\sigma^{2}\left(s-s_{0}\right) V-\frac{d}{d s}\left(\frac{\Sigma_{0}}{V_{0}+1}\right) V-\frac{\Sigma_{0}}{V_{0}+1}\left(D+\left(s-s_{0}\right) \Sigma\right)=\left(\begin{array}{c}
\frac{d}{d s}\left(\frac{\Sigma_{0}}{V_{0}+1}\right) \\
\overrightarrow{0}
\end{array}\right),
$$


The scalar product of this equality with respect to $V, D, \Sigma$, gives, respectively

$$
\begin{gathered}
-\sigma^{2}\left(s-s_{0}\right)+\frac{d}{d s}\left(\frac{\Sigma_{0}}{V_{0}+1}\right)=-\frac{d}{d s}\left(\frac{\Sigma_{0}}{V_{0}+1}\right) V_{0}, \\
-\frac{\Sigma_{0}}{V_{0}+1} \beta^{2}=-\frac{d}{d s}\left(\frac{\Sigma_{0}}{V_{0}+1}\right) D_{0} \\
-\frac{\Sigma_{0}}{V_{0}+1} \sigma^{2}\left(s-s_{0}\right)=-\frac{d}{d s}\left(\frac{\Sigma_{0}}{V_{0}+1}\right) \Sigma_{0}
\end{gathered}
$$

with $D=\left(D_{0}, \vec{D}\right)$. The content of IV.16 and IV.18 is the same: $\frac{d}{d s}\left(\frac{\Sigma_{0}}{V_{0}+1}\right)=$ $\frac{\sigma^{2}\left(s-s_{0}\right)}{V_{0}+1}$. Injecting this equality in IV.17 we obtain

$$
\Sigma_{0} \beta^{2}=\sigma^{2}\left(s-s_{0}\right) D_{0}
$$

Next, taking into account that, from IV.6, $\dot{\Sigma}_{0}=\sigma^{2}\left(s-s_{0}\right) V_{0}$ and $\dot{D}_{0}=\beta^{2} V_{0}$, we can compute the proper time derivative of $(\overline{I V .19})$, which yields $D_{0}=0$. This result implies that $\beta=0$ (since $\dot{D}_{0}=\beta^{2} V_{0}$ and $V_{0}>0$ ).

We conclude that, within the vanishing Snap case, the requirement of constant proper Jerk corresponds to the case $\beta=0$, analyzed in subsection IVA, which also implies that the motion is $2 \mathrm{~d}$.

\section{In Minkowski spacetime:}

A trajectory of vanishing Snap has constant proper Jerk if and only if the parameter $\beta$ in IV.1 vanishes. Then the motion takes place in a plane (2d).

Note however that constant proper Jerk trajectories do not need to be in a plane. As a matter of fact, the equation for constant proper Jerk (IV.14 can be equivalently written as

$$
\vec{\Sigma}-\frac{(\vec{b}, \vec{V})}{V_{0}+1} \vec{V}=\vec{b}_{0}
$$

where we have used that $(\Sigma, V)=0$ implies $\Sigma_{0}=\frac{(\vec{\Sigma}, \vec{V})}{V_{0}}$ which, together with $($ IV.14), gives $\Sigma_{0}=\vec{b}_{0} \cdot \vec{V}$. Also from IV.20 one can go back to IV.14. Since IV.20 is a second order -non linear- differential vector equation in normal form

$$
\ddot{\vec{V}}-(A, A) \vec{V}-\frac{(\vec{b}, \vec{V})}{V_{0}+1} \vec{V}-\vec{b}_{0}=0
$$

with $(A, A)=-A_{0}^{2}+(\vec{A})^{2}=-\left(\frac{(\dot{\vec{V}}, \vec{V})}{V_{0}}\right)^{2}+(\dot{\vec{V}})^{2}$, we infer that it has solutions for arbitrary initial conditions $\vec{V}\left(t_{0}\right)$ and $\dot{\vec{V}}\left(t_{0}\right)$. Thus the solution trajectories of IV.21) are not restricted to be in a plane in Minkowski spacetime. 


\section{Some equivalences.}

Our findings can be summed up with the following equivalences. Let us introduce the three sentences:

A: The trajectory has vanishing Snap,

B: The trajectory has constant proper Jerk,

C: The trajectory takes place in a plane (2d) in Minkowski spacetime,

then what we have shown in subsection IV C is that

1) $\mathbf{A} \cap \mathbf{B} \Rightarrow \mathbf{C}$.

We can also show that

2) $\mathrm{B} \cap \mathrm{C} \Rightarrow \mathrm{A}$,

and that

3) $\mathrm{A} \cap \mathrm{C} \Rightarrow \mathrm{B}$.

The proofs of 2) and 3) are postponed to section VII in which we show that in 2d, the notions of vanishing Snap and constant proper Jerk coincide. See in particular the equations (VII.1) and (VII.3) which prove the more general statement that in $2 \mathrm{~d}$ the notion of vanishing $(n+1)$-acceleration and constant proper $n$-acceleration coincide.

Summarizing:

In Minkowski spacetime:

$\mathbf{A} \cap \mathbf{B}=\mathbf{B} \cap \mathbf{C}=\mathbf{A} \cap \mathbf{C}=\mathbf{A} \cap \mathbf{B} \cap \mathbf{C}$.

\section{THE VIEW FROM ANOTHER FRAME OF REFERENCE}

Consider a motion with constant proper acceleration, that is, satisfying (II.2) (with constant $\vec{a}$ ) or, equivalently, (II.7) in a given frame of reference. If our Minkowski spacetime has dimension $>2$, we may wonder how this motion is seen from a different frame of reference, and in particular what will be the proper acceleration in the instantaneous rest frame of the observer.

So consider a general $2 \mathrm{~d}$ motion in a $4 \mathrm{~d}$ (coordinates $t, x, y, z)$ Minkowski spacetime although $3 \mathrm{~d}$ is enough to see what happens. Choose coordinates so that this rectilinear motion takes place in the $t, x$ coordinates, $V=\left(V_{0}, \vec{V}\right)=\left(V_{0}(s), V_{1}(s), 0,0\right)$, and let us apply to it a proper boost $G$ characterized by sending a given timelike unit-norm constant vector $U=\left(U_{0}, \vec{U}\right)=\left(U_{0}, U_{1}, U_{2}, 0\right)$ to rest, $(1,0,0,0)$, that is,

$$
G=\left(\begin{array}{cc}
U_{0} & -\vec{U} \\
-\vec{U} & I+\frac{1}{U_{0}+1} \vec{U} \otimes \vec{U}
\end{array}\right)
$$


After this change of reference frame, the new velocity of the observer will be $W(s)=: G V(s)$ and there will be an instantaneous proper boost $B_{W}(s)^{4}$ which sends this velocity to the instantaneous rest frame, $B_{W}(s) W(s)=B_{W}(s) G V(s)=(1,0,0,0)=V_{p}$. But we know that $B(s) V(s)=(1,0,0,0)=V_{p}$. So $B_{W}(s) G B(s)^{-1}$ must be a spatial rotation $R(\theta(s))$,

$$
B_{W}(s) G B(s)^{-1} \equiv R(s)=\left(\begin{array}{cc}
1 & 0 \\
0 & R_{i j}(\theta(s))
\end{array}\right) .
$$

This rotation acts on the 2-dimensional plane $\langle x, y\rangle$ and has $\cos (\theta)$ determined as

$$
\cos (\theta(s))=1-\frac{\left(U_{2}\right)^{2}\left(V_{0}-1\right)}{\left(U_{0}+1\right)(1-(U \cdot V))},
$$

with $U \cdot V=-U_{0} V_{0}+\vec{U} \cdot \vec{V}$. The axis of rotation of $(\mathrm{V} .1)$ is in the direction $\vec{U} \wedge \vec{V}$.

Note that Eq. V.2 is symmetric with respect to $U$ and $V$. Indeed, since

$$
\left(U_{2}\right)^{2}=\frac{|\vec{U} \wedge \vec{V}|^{2}}{|\vec{V}|^{2}}=|\vec{U}|^{2}(\sin \alpha)^{2}=\left(U_{0}^{2}-1\right)(\sin \alpha)^{2},
$$

where $\alpha$ is the angle between $\vec{V}$ and $\vec{U}$, then, in terms of $\frac{\theta}{2}$ equation $\mathrm{V} .2$ becomes

$$
\left(\sin \frac{\theta}{2}\right)^{2}=\left(\frac{\left(U_{0}-1\right)\left(V_{0}-1\right)}{2(1-(U \cdot V))}\right)(\sin \alpha)^{2},
$$

(note that there is an $\alpha$ dependence in $(U \cdot V)$ as well) and thus the rotation $R_{i j}(\theta)$ becomes the identity only for $\sin \alpha=0$, which is equivalent to the vanishing of $U_{2}$-when $\vec{U}$ and $\vec{V}$ are parallel.

When the rotation is not the identity it can still be a constant rotation (that is, $s$ independent) only if $V_{1}$ is constant, that is, when the rectilinear motion of the observer is uniform. But not for the accelerated observer.

Note on the other hand that the proper acceleration, as seen from the new reference frame, is ([p] is for "proper"), $A_{W}^{[p]}=B_{W}(s) A_{W}=B_{W}(s) G A=B_{W}(s) G B(s)^{-1} A^{[p]}=R(\theta(s)) A^{[p]}$. So for a constant proper acceleration $A^{[p]}=\left(0, a_{0}, 0,0\right)$, we end up with

$$
A_{p}(s)=\left(\begin{array}{c}
0 \\
a_{0} \\
0 \\
0
\end{array}\right) \longrightarrow A_{W}^{[p]}=a_{0}\left(\begin{array}{c}
0 \\
\cos (\theta(s)) \\
\sin (\theta(s)) \\
0
\end{array}\right)
$$

Thus, if we are not in the case of linear uniform motion for the observer, only when the component $U_{2}$ vanishes will this rotation be independent of the component $V_{1}(s)$, in

\footnotetext{
${ }^{4} B_{W}(s)$ is just the proper boost $(\mathrm{IV} .12$ with $V$ replaced by $W$.
} 
which case the rotation becomes the identity matrix. The boost $G$ in this case will be along the $x$ direction, thus commuting with the family of boosts $B(s)$, which are already in such direction.

This argument applies as well to any motion (not necessarily $2 \mathrm{~d}$ ) with constant proper Jerk, or constant proper Snap, etc. The eventual constancy of these objects is frame dependent and only for changes of frame engendered by the boost $G$ when it points in the direction of the 3 -velocity of the observer (boost in the 2-dimensional plane $\langle t, x\rangle$ in our case), will this constancy be preserved. Indeed, we should rephrase the notion of constant proper Jerk as: a trajectory has constant proper Jerk in Minkowski spacetime if there exists a frame of reference such that equation IV.14 holds for some constant vector $\vec{b}_{0}$.

The conclusion is clear and general: the notion of constant proper acceleration, constant proper Jerk, constant proper Snap, etc, as defined in (IV.13) for the Jerk, and trivially generalized to all these objects, is not invariant under changes of reference frame. On the other hand, it is reasonable to consider that the "constancy" of some $A_{n}$ should be associated with the vanishing of the next $A_{n+1}$. For that we need the additional requirement (see section IV D for the case of the constant proper Jerk) that the trajectory takes place in a $2 \mathrm{~d}$ plane in Minkowski spacetime.

All in all, and in view to eventual generalizations to curved spacetime, our statements should at least be invariant under changes of frame of reference, with the expectation for them to generalize to statements invariant under diffeomorphisms in generally covariant theories. If we want to make invariant statements we should rely in the curvature invariants associated with the trajectory. These curvature invariants will be introduced in the context of the Frenet-Serret formalism. This formalism and its relation with the objects introduced so far (Jerk, Snap, etc.), is discussed in the next section.

\section{THE FRENET-SERRET FRAMEWORK}

The Lorentzian setting for the originally Euclidean Frenet-Serret formalism was studied in [8]. Here we directly adapt to the Lorentzian setting the Euclidean construction in [9]. Consider a trajectory $X(s)$ parametrized by the proper time $s$, so that the velocity vector $U_{0}$, defined as $U_{0} \equiv V=\frac{d}{d s} X$, has $U_{0}^{2}=-1$.

The first curvature invariant (also known as scalar curvature) is defined by $\kappa_{1}=\left|\frac{d}{d s} U_{0}\right|$, and the vector $U_{1}$, orthogonal to $U_{0}$-and hence spacelike- and normalized, is defined as,

$$
U_{1}=\frac{1}{\kappa_{1}} \frac{d}{d s} U_{0}, \quad U_{1}^{2}=1
$$

Of course this construction only works for non-vanishing $\kappa_{1}$. There may be exceptions at some isolated points in the trajectory, but if $\kappa_{1}=0$ for an entire segment of the trajectory, 
then the construction finishes here -for this segment. Note that the acceleration $A$ defined in previous sections (see (II.4), (III.1)) is just $A=\kappa_{1} U_{1}\left(\right.$ so $\left.|A|=\kappa_{1}\right)$.

Next, noticing that the vector $\frac{d}{d s} U_{1}-\kappa_{1} U_{0}$ is orthogonal to both $U_{0}$ and $U_{1}$, we define the second curvature invariant (also known as the torsion scalar)

$$
\kappa_{2}=\left|\frac{d}{d s} U_{1}-\kappa_{1} U_{0}\right|
$$

and as long as $\kappa_{2}>0$ (otherwise the construction finishes here) we define the spacelike normalized vector $U_{2}$ as

$$
U_{2}=\frac{1}{\kappa_{2}}\left(\frac{d}{d s} U_{1}-\kappa_{1} U_{0}\right)
$$

which is an expression that can be read also as

$$
\frac{d}{d s} U_{1}=\kappa_{2} U_{2}+\kappa_{1} U_{0}, \quad U_{2}^{2}=1,\left(U_{1} \cdot U_{2}\right)=0,\left(U_{0} \cdot U_{2}\right)=0 .
$$

Again, having noticed that the vector $\frac{d}{d s} U_{2}+\kappa_{2} U_{1}$ is orthogonal to $U_{0}, U_{1}$ and $U_{2}$, we define the third curvature invariant (also known as the hypertorsion scalar)

$$
\kappa_{3}=\left|\frac{d}{d s} U_{2}+\kappa_{2} U_{1}\right|
$$

alongside with the definition of $U_{3}$ (as long as $\kappa_{3}>0$, otherwise the construction finishes here)

$$
U_{3}=\frac{1}{\kappa_{3}}\left(\frac{d}{d s} U_{2}+\kappa_{2} U_{1}\right)
$$

which is an expression that can be read also as

$$
\frac{d}{d s} U_{2}=\kappa_{3} U_{3}-\kappa_{2} U_{1}, \quad U_{3}^{2}=1,\left(U_{2} \cdot U_{3}\right)=0,\left(U_{1} \cdot U_{3}\right)=0,\left(U_{0} \cdot U_{3}\right)=0 .
$$

and this construction continues as long as it is allowed by the number of spacetime of dimensions and the specific curvatures of the trajectory considered. In $4 \mathrm{~d}$ Minkowski we have reached the full constuction.

Note that these curvature invariants, although constructed for trajectories in Minkowski spacetime, can be generalized to curved spacetimes along the lines of section IIE. In this broader sense, these curvature invariants behave as scalars under target space diffeomorphisms (coordinate reparametrizations) and as invariants under world line diffeomorphisms (reparametrizations of the evolutionary parameter of the trajectory). The derivatives of these curvature invariants with respect to the proper time are also curvature invariants with the same properties with respect to both types of diffeomorphisms as the original curvature invariants discussed above. 


\section{A. Revisiting the constantly accelerated observer}

By the reasons given in section $(\mathrm{V})$. the solution given in section II C for the $3 \mathrm{~d}$ constantly accelerated observer does not show constant proper acceleration when examined form another generic frame of reference. As said before, this is not a real problem for the claim that this is a solution for the trajectory of a constantly accelerated observer, but we can take advantage of the Frenet-Serret formalism of section (VI) to show that it is possible to characterize this solution in an invariant way.

One can easily compute the first and second curvature scalars for the solution (II.16). By construction we clearly have $\kappa_{1}=a_{0}$. The second curvature scalar turns out to be $\kappa_{2}=a_{0} \sqrt{1-d}$ (Thus $\kappa_{2}<\kappa_{1}$ ). Since we are in 3d Minkowski spacetime, $\kappa_{3}$ vanishes. With these data we can reconstruct the solution (II.16), as we do now.

For constant values of $\kappa_{1}, \kappa_{2}, \kappa_{3}$, the solution trajectories were classified in [8]. Using the Frenet Serret equations of section (VI), being $\kappa_{1}$ and $\kappa_{2}$ constants (and $\kappa_{3}=0$ ), we obtain the equation $\left(U_{0}=V\right)$

$$
\dddot{V}=\left(\kappa_{1}^{2}-\kappa_{2}^{2}\right) \dot{V},
$$

and in our case $\kappa_{1}^{2}-\kappa_{2}^{2}=a_{0}^{2} d>0$. The fact that $\kappa_{2}<\kappa_{1}$ is crucial. The general solution for $V$ is then of the form

$$
V=M \cosh \left(\sqrt{d} a_{0}\left(s-s_{0}\right)\right)+N \sinh \left(\sqrt{d} a_{0}\left(s-s_{0}\right)\right)+G,
$$

with $M, N, G$ constant vectors to be determined. The condition $(V)^{2}=-1$ imposes $(M, N)=(M, G)=(N, G)=0,(N)^{2}=-(M)^{2}$ and $(M)^{2}+(G)^{2}=-1$. The acceleration is

$$
A=\dot{V}=\sqrt{d} a_{0}\left(M \sinh \left(\sqrt{d} a_{0}\left(s-s_{0}\right)\right)+N \cosh \left(\sqrt{d} a_{0}\left(s-s_{0}\right)\right)\right),
$$

and $(A)^{2}=a_{0}^{2}$ imposes $(M)^{2}=-\frac{1}{d}$ which implies $(N)^{2}=\frac{1}{d}$ and $(G)^{2}=\frac{1-d}{d}$.

With all these restrictions we can choose $M, N, G$. For instance the choice made in (II.16) is

$$
M=\left(\begin{array}{c}
\frac{1}{d} \\
0 \\
\frac{\sqrt{1-d}}{d}
\end{array}\right), \quad N=\left(\begin{array}{c}
0 \\
\frac{1}{\sqrt{d}} \\
0
\end{array}\right), \quad G=\left(\begin{array}{c}
\frac{1-d}{d} \\
0 \\
\frac{\sqrt{1-d}}{d}
\end{array}\right) .
$$

Another, simpler choice, related to the one above by a boost, is

$$
M=\left(\begin{array}{c}
\frac{1}{\sqrt{d}} \\
0 \\
0
\end{array}\right), \quad N=\left(\begin{array}{c}
0 \\
\frac{1}{\sqrt{d}} \\
0
\end{array}\right), \quad G=\left(\begin{array}{c}
0 \\
0 \\
\sqrt{\frac{1-d}{d}}
\end{array}\right)
$$


with which the solution (II.16) takes the form

$$
V=\left(\begin{array}{c}
V_{0} \\
\vec{V}
\end{array}\right)=\left(\begin{array}{c}
\frac{1}{\sqrt{d}} \cosh \left(\sqrt{d} a_{0}\left(s-s_{0}\right)\right) \\
\frac{1}{\sqrt{d}} \sinh \left(\sqrt{d} a_{0}\left(s-s_{0}\right)\right) \\
\sqrt{\frac{1-d}{d}}
\end{array}\right) .
$$

Interestingly we may interpret it as the standard accelerated motion along the $x$ direction together with a motion along the $y$ direction which is linear in the proper time. Note however that the acceleration $A$ associated with this solution (VI.2) does not give a constant proper acceleration, whereas the solution (II.16) does, because of the frame dependence explained in section V. But we can nevertheless still claim that (VI.2) describes a motion with constant proper acceleration.

Since the coordinate time satisfies $\frac{d t}{d s}=V_{0}=\frac{1}{\sqrt{d}} \cosh \left(\sqrt{d} a_{0}\left(s-s_{0}\right)\right)$, we can set $t=$ $\frac{1}{a_{0} d} \sinh \left(\sqrt{d} a_{0}\left(s-s_{0}\right)\right.$ ) (so $s=s_{0}$ is $\left.t=0\right)$ and the position vector becomes, in terms of the coordinate time,

$$
X=X(0)+\left(\begin{array}{c}
t \\
\frac{1}{a_{0} d}\left(\sqrt{1+a_{0}^{2} d^{2} t^{2}}-1\right) \\
\frac{\sqrt{1-d}}{a_{0} d} \operatorname{arcsinh}\left(d a_{0} t\right)
\end{array}\right)
$$

We notice that this reconstruction relies on the fact that, in addition to the constancy of $\kappa_{1}, \kappa_{2}$, the inequality $\kappa_{2}<\kappa_{1}$ is satisfied. We may state

In Minkowski spacetime (any dimension), the observer with constant proper acceleration is characterized by $\kappa_{3}=0$ and $\kappa_{1}, \kappa_{2}$ constants with $\kappa_{2}<\kappa_{1}$. The particular case $\kappa_{2}=0$ corresponds to motion in a plane and it also identifies the case of the vanishing of the Jerk.

These statements can be exported as definitions in curved spacetime, just by dropping the reference to the trajectory taking place in a plane,

In curved spacetime, the observer with constant proper acceleration is defined by $\kappa_{3}=0$ and $\kappa_{1}, \kappa_{2}$ constants with $\kappa_{2}<\kappa_{1}$. The particular case $\kappa_{2}=0$ corresponds to the vanishing of the Jerk.

Although both solutions, (II.16) and (VI.2), are related by a simple boost, it is worth to notice that the trajectory (VI.3), unlike (II.17), does not have asymptotes in the (x,y) plane. Now the ingoing and outgoing directions in the 2-space are just oposite: $(-1,0),(1,0)$. The parameter $d$ is now associated with the ratio of the two space coordinates at infinity. Indeed we have, for (VI.3), $(X=\{t, x, y\})$,

$$
\lim _{s \rightarrow \infty} \frac{y}{\log x}=\frac{\sqrt{1-d}}{a_{0}^{2} d} .
$$


For the sake of completeness we may consider the cases with $\kappa_{2} \geq \kappa_{1}$. In view of VI.1, the case $\kappa_{2}=\kappa_{1}$ is immediate. The case with $\kappa_{2}>\kappa_{1}$ corresponds to uniform circular motion, which we review briefly in the Appendix $\mathrm{A}$ and give expressions for its $n$-accelerations.

\section{B. Jerk and Snap in terms of the Frenet-Serret basis}

In terms of the Frenet-Serret basis, we can write the Jerk and Snap as

$$
\begin{gathered}
\Sigma=\frac{d \kappa_{1}}{d s} U_{1}+\kappa_{1} \kappa_{2} U_{2} \\
\Xi=\left(\frac{d^{2} \kappa_{1}}{d s^{2}}-\kappa_{1} \kappa_{2}^{2}\right) U_{1}+\left(\frac{d}{d s}\left(\kappa_{1}^{2} \kappa_{2}\right)\right) U_{2}+\left(\kappa_{1} \kappa_{2} \kappa_{3}\right) U_{3} .
\end{gathered}
$$

Now we are ready to reproduce the results of sections II D and IV.

Assuming that the acceleration is non vanishing, $\kappa_{1}>0$ (otherwise the Jerk already vanishes), vanishing of the Jerk $(\Sigma=0)$, means $\kappa_{2}=0$ and $\frac{d \kappa_{1}}{d s}=0$. Here we recover the results of sction IID. Whereas $\kappa_{2}=0$ signals that the trajectories lie in a plane, the second condition, integrated to $\kappa_{1}=|A|=$ constant, is the well know equation (II.11) for the constantly accelerated observer (hyperbolic trajectories) in Minkowski $2 \mathrm{~d}$.

Vanishing of the Snap is equivalent to

$$
\Xi=0 \quad \Leftrightarrow \quad \kappa_{3}=0, \quad \kappa_{1}^{2} \kappa_{2}=\text { constant }, \quad \frac{d^{2} \kappa_{1}}{d s^{2}}-\kappa_{1} \kappa_{2}^{2}=0
$$

Solving these equations we obtain for $\kappa_{1}, \kappa_{2}$, in terms of the notation of section IV,

$$
\kappa_{1}=\sqrt{\sigma^{2}\left(s-s_{0}\right)^{2}+\beta^{2}}, \quad \kappa_{2}=\frac{\beta \sigma}{\sigma^{2}\left(s-s_{0}\right)^{2}+\beta^{2}} .
$$

with $\sigma>0, \beta \geq 0$ (for $\sigma=0$ we are back to the vanishing Jerk case). In terms of the orthogonal vectors $V, D, \Sigma$, used in section IV, we have $\left(U_{2}\right.$ only exists if $\left.\beta>0\right)$,

$$
U_{0}=V, \quad U_{1}=\frac{1}{\kappa_{1}}\left(D+\left(s-s_{0}\right) \Sigma\right), \quad U_{2}=\frac{1}{\beta \sigma \kappa_{1}}\left(-\sigma^{2}\left(s-s_{0}\right) D+\beta^{2} \Sigma\right) .
$$

Notice from VI.6 that

$$
\kappa_{2}=0 \Leftrightarrow \beta=0
$$

This is the invariant statement we were after: we showed in section [V] that within the vanishing Snap condition, constant proper Jerk was equivalent to $\beta=0$. On the other hand, the constancy of the proper Jerk does not imply the vanishing of the Snap.

We are led to the following statement 
In Minkowski spacetime:
A trajectory of vanishing Snap has constant proper Jerk only if $\kappa_{2}=0$ (thus
implying $\kappa_{i}=0, i>2$ ) and $\frac{d^{2} \kappa_{1}}{d s^{2}}=0$. This trajectory is therefore $2 d$ and $\kappa_{1}$
is (the absolute value of) an arbitrary first degree polynomial in the proper
time.

This satement can be taken as a definition in curved spacetime

$$
\begin{aligned}
& \text { In curved spacetime: } \\
& \text { A trajectory of vanishing Snap and constant proper Jerk is characterized by } \\
& \kappa_{2}=0 \text { and } \frac{d^{2} \kappa_{1}}{d s^{2}}=0 .
\end{aligned}
$$

Let us take stock of our results so far. We have seen that for $n=1$ and $n=2$, the concepts of constant $n$-acceleration and vanishing $(n+1)$-acceleration only coincide if the motion is $2 \mathrm{~d}$ in Minkowski spacetime or, more generally, either in flat or curved spacetime, if $\kappa_{2}=0$. On the other hand we will see in section VII that if $\kappa_{2}$ vanishes then both concepts coincide $\forall n$. It is plausible that, as in the $n=1$ and $n=2$ cases, this coincidence only happens when $\kappa_{2}=0$.

In the case of vanishing Snap, the fact that the curvature invariants are no longer constants is the circumstance that makes it difficult to find analytic solutions. In addition, we do not have analytic solutions for the constant proper Jerk case nor for its characterization through curvature invariants.

Up to now, we have shown that the first, second and third accelerations $A_{1}=A, A_{2}=$ $\Sigma, A_{3}=\Xi$, have a common structure, displayed below. It is not difficult to see that this common structure is shared by the whole family of $n$-accelerations, $A_{n}$,

$$
A_{n+1}=\kappa_{1}^{(n)} U_{1}+\text { terms vanishing for } \kappa_{2}=0
$$

where $\kappa_{1}^{(n)}=\frac{d^{n}}{d s^{n}} \kappa_{1}$.

Considering this structure, and anticipating the results of section VII, we may state that

In flat or curved spacetime:

If the second curvature invariant vanishes, $\kappa_{2}=0$, then the concepts of vanishing $(n+1)$-acceleration and constant proper $n$-acceleration are equivalent and are characterized by $\kappa_{1}^{(n)}=0$. This trajectory is therefore $2 d$ and $\kappa_{1}$ is (the absolute value of) an arbitrary $(n-1)$-degree polynomial in the proper time. 


\section{2D MINKOWSKI: CONSTANT PROPER JERK, SNAP, CRACKLE...}

In this section we will obtain the solutions for the constant proper $n$-acceleration under the assumption that the $(n+1)$-acceleration vanishes. Since we have shown in the previous section that in this case the trajectories are 2-dimensional, we will work in a 2-dimensional Minkowski spacetime.

In a $2 \mathrm{~d}$ Minkowski spacetime we can easily generalize the results in (IV.4). As stated in section III, we use the notation $V$ for the velocity (normalized to $(V)^{2}=-1$ ), $A_{1}$ for the acceleration $A ; A_{2}$ for the 2-acceleration or Jerk, $\Sigma ; A_{3}$ for the 3-acceleration or Snap, $\Xi$; etc.

With the general parametrization

$$
V(s)=\left(\begin{array}{c}
\cosh (f(s)) \\
\sinh (f(s))
\end{array}\right)
$$

we obtain

$$
A_{1}(s)=f^{\prime}(s)\left(\begin{array}{c}
\sinh (f(s)) \\
\cosh (f(s))
\end{array}\right), \quad A_{2}(s)=f^{\prime \prime}(s)\left(\begin{array}{c}
\sinh (f(s)) \\
\cosh (f(s))
\end{array}\right),
$$

(note that $\kappa_{1}=\left|f^{\prime}(s)\right|$ ) and in general,

$$
A_{n}(s)=f^{(n)}(s)\left(\begin{array}{c}
\sinh (f(s)) \\
\cosh (f(s))
\end{array}\right)
$$

that is, $\left|\kappa_{1}^{(n-1)}(s)\right|=\left|f^{(n)}(s)\right|$. All this information can be examined at any proper time $s$ from the instantaneous rest system of the observer, through the boost

$$
B(s)=\left(\begin{array}{cc}
\cosh (f(s)) & -\sinh (f(s)) \\
-\sinh (f(s)) & \cosh (f(s))
\end{array}\right), .
$$

which sends $V$ to the rest frame -at this proper time $s$ - and sends $A_{1}$ to the proper acceleration, $A_{2}$ to the proper Jerk and in general sends $A_{n}$ to its proper expression $A_{n}^{[p]}$ ([p] is for "proper") in the rest frame, $A_{n}(s) \rightarrow B(s) A_{n}(s)=A_{n}^{[p]}(s)$. Thus we have

$$
V^{[p]}(s)=\left(\begin{array}{l}
1 \\
0
\end{array}\right), \quad A_{n}^{[p]}(s)=f^{(n)}(s)\left(\begin{array}{l}
0 \\
1
\end{array}\right) \text { for } n>1 .
$$

We conclude that in $2 \mathrm{~d}$ Minkowski, a constant proper $A_{n}^{[p]}$ corresponds to $f$ being a $n$-degree polynomial $f(s)=P_{n}(s)$ and it also corresponds to a vanishing $A_{(n+1)}$.

$$
\begin{aligned}
& \text { In } 2 d \text { Minkowski, constant proper } A_{n}^{[p]} \Leftrightarrow \text { vanishing } A_{(n+1)} \\
& \Leftrightarrow f(s)=P_{n}(s) \Leftrightarrow \kappa_{1}=\left|P_{n}^{\prime}(s)\right|
\end{aligned}
$$


The fact that $f(s)$ is a $n$-degree polynomial in proper time was already obtained in [5]. Now we provide analytic solutions for the trajectories in the simplest case $P_{n}(s)=\frac{s^{n}}{s_{0}^{n}}$. This case captures the asymptotic behaviour at $s \rightarrow \pm \infty$. The trajectories are

$$
X(s)=\left(\begin{array}{c}
X_{0}(s) \\
X_{1}(s)
\end{array}\right) \text {. }
$$

with

$$
\begin{gathered}
X_{0}(s)=s_{1} F_{2}\left(\frac{1}{2 n} ; \frac{1}{2}, 1+\frac{1}{2 n} ; \frac{1}{4}\left(\frac{s}{s_{0}}\right)^{2 n}\right), \\
X_{1}(s)=\frac{1}{n+1} s\left(\frac{s}{s_{0}}\right)^{n}{ }_{1} F_{2}\left(\frac{1}{2}+\frac{1}{2 n} ; \frac{3}{2}, \frac{3}{2}+\frac{1}{2 n} ; \frac{1}{4}\left(\frac{s}{s_{0}}\right)^{2 n}\right),
\end{gathered}
$$

where ${ }_{1} F_{2}$ is the generalized hypergeometric function,

$$
{ }_{1} F_{2}(a ; b, c ; z)=\sum_{k=0}^{\infty} \frac{(a)_{k}}{(b)_{k}(c)_{k}} \frac{z^{k}}{k !}, \quad(x)_{k}:=\frac{\Gamma(x+k)}{\Gamma(x)},
$$

with $\Gamma$ the Gamma function.

To depict the trajectories as tangent to the future light cone with vertex at the origin of coordinates we must make a translation:

$$
X_{1}(s) \rightarrow X_{1}(s)+\Gamma\left(\frac{n+1}{n}\right) s_{0}=: \bar{X}_{1}(s) .
$$

This quantity, $\Gamma\left(\frac{n+1}{n}\right) s_{0}$, is the equal-time distance between the observer at $t=0$ and the origin of coordinates. This origin of coordinates belongs to the light cone line defining the horizon for the observer.

Still keeping with $P_{n}(s)=\frac{s^{n}}{s_{0}^{n}}$, if we compare the -infinite- time of travel, from past infinity $s \rightarrow-\infty$ to future infinity $s \rightarrow \infty$, of the trajectory corresponding to $P_{n}(s)$ and that of the light (considering in the $n=$ odd case that the light is reflected at the origin of coordinates), we find a finite difference $d_{n}$. This time delay is

$$
d_{n}=2 \Gamma\left(\frac{n+1}{n}\right) s_{0} .
$$

It is maximum for $n=1, d_{1}=2 s_{0}$, that is, for the constantly accelerated observer, whereas the minimum is reached for $n=2, d_{2}=2 \Gamma\left(\frac{3}{2}\right) s_{0}$. After this minimum, $d_{n}$ increases monotonically with $n$, reaching $d_{1}$ in the limit $n \rightarrow \infty$.

Here we depict trajectories for $n=1$, constant proper acceleration (Fig 1); $n=2$, constant proper Jerk (Fig 2); and $n=3$, constant proper Snap (Fig 3).

In general one may consider, for an arbitrary polynomial $P_{n}(s)$ with real coefficients, its real roots, $s_{k}$. These roots are associated with the points in the trajectory $\left(X_{0}\left(s_{k}\right), \bar{X}_{1}\left(s_{k}\right)\right)$ with vanishing 3 -velocity. If the root is simple or its degeneracly is an odd number, then the 3-velocity changes sign at this point, and graphically we find a turning point in the trajectory, like the four turning points depicted in Fig (4). 


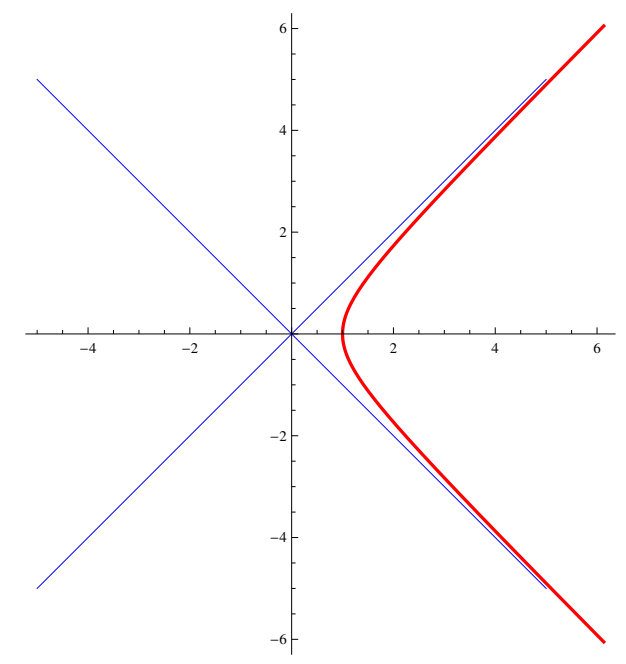

FIG. 1: 2d Minkowski. $n=1, P_{1}(s)=s$. The well known hyperbola depicting the trajectory of the observer with vanishing Jerk $=$ constant proper acceleration. The line $x=t$ of the light cone is a horizon for the observer. This horizon appears in all the cases $n \geq 1$. Notice that, as it will happen with all $n=$ odd cases, the trajectory in the space coordinate $x$ goes from $+\infty$ in the past to $+\infty$ in the future, reaching the minimum at $t=0$ (in our parametrization).

\section{CONCLUSIONS}

Starting within the context of Minkoswki spacetime and later generalizing to curved spacetime, we characterize the concept of a trajectory with constant proper acceleration by moving to the instantaneous comoving frame of reference of the observer, and choosing the boost that sends the observer to rest as a pure boost, with no additional rotation attached. We find that the motion of such an observer is at most three dimensional -including the time coordinate. Our characterization is formulated in the following way: "An observer undergoes a motion with constant proper acceleration if there exist an intertial frame of reference such that, using it for the description of the trajectory, the pure boost that sends the observer to rest is also sending the acceleration vector to a constant vector". Formulated in such a way, this sentence does not seem to be exportable to curved spacetime. At this point the Frenet Serret formalism comes to the rescue and we show that our definition is equivalent to the statement that the third curvature invariant $\kappa_{3}$ vanishes $(\Rightarrow 3 \mathrm{~d}$ motion in Minkoswki spacetime), that the first and second curvature invariants $\kappa_{1}$ and $\kappa_{2}$ are constant and that $\kappa_{2}<\kappa_{1}$. In fact when $\kappa_{2}$ vanishes the motion is $2 \mathrm{~d}$ and it is the standard textbook motion of the constantly accelerated observer in Minkoswki spacetime. This invariant characterization is immediately exported to curved spacetime as the definition of the observer with constant proper acceleration. Our definition excludes the uniform circular motion (see Appendix A), for which $\kappa_{2}>\kappa_{1}$, with both $\kappa_{2}$ and $\kappa_{1}$ constants. 


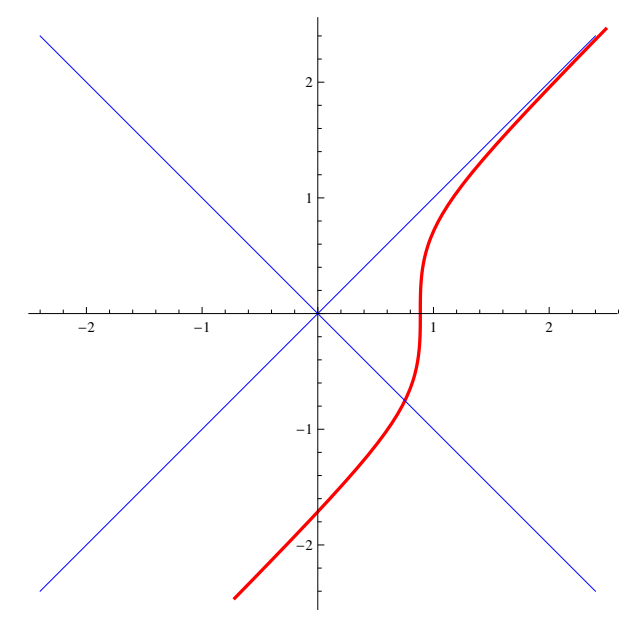

FIG. 2: $2 d$ Minkowski. $n=2, P_{2}(s)=s^{2}$. The trajectory of the observer with vanishing Snap = constant proper Jerk. Again, the line $x=t$ of the light cone is a horizon for the observer. Notice that, as it will happen with all $n=$ even cases, the trajectory goes from $-\infty$ in the past to $+\infty$ in the future.

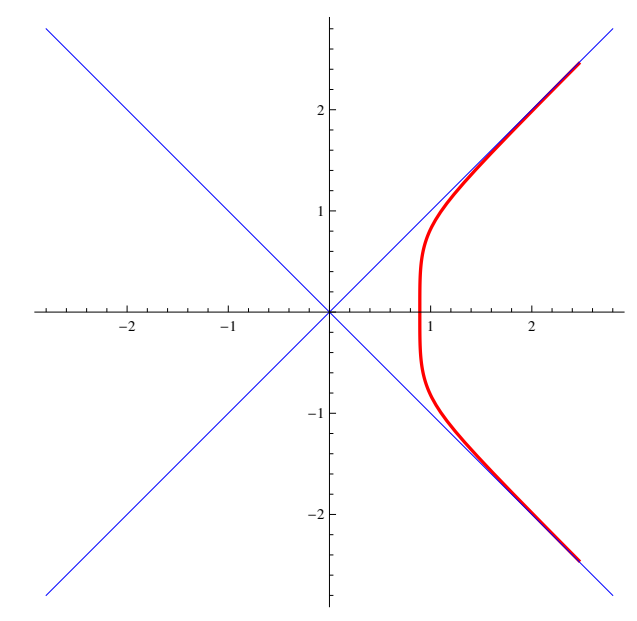

FIG. 3: 2d Minkowski. $n=3, P_{3}(s)=s^{3}$. The trajectory of the observer with vanishing Crackle $=$ constant proper Snap.

Let us comment that our definition is much more restrictive than that used in [6], inspired in [11]. In [6] the observer with constant proper acceleration (uniform acceleration) is defined by the constancy of the first, second and third curvature invariants (in a 4 dimensional Minkoswki spacetime), and it is understood that in higher dimensions the definition will include the constancy of the new curvature invariants that may appear. Of course it is all a matter of definition, but we would like to point out that with our definition, for whatever dimension of the Minkowski spacetime, there is always a frame of reference in which the motion will at most be three dimensional $\left(\kappa_{3}=0\right)$. 


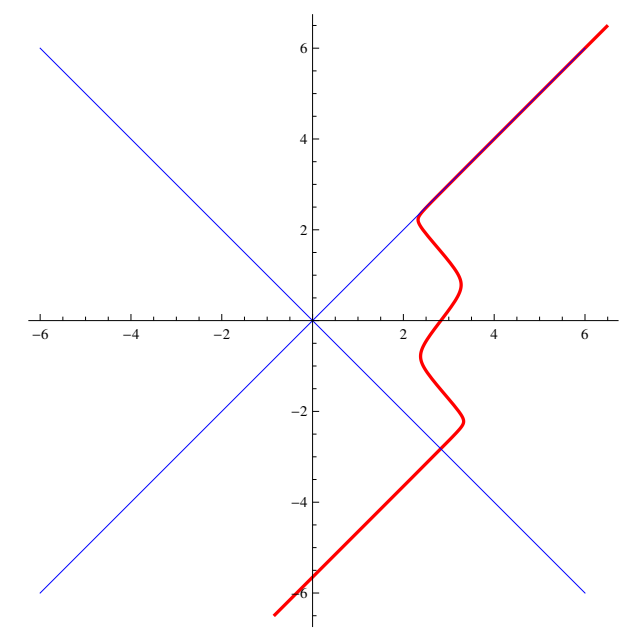

FIG. 4: 2d Minkowski. $n=4, P_{4}(s)=s^{4}-3 s^{2}+1$. Now the trajectory of the observer with vanishing Pop $=$ constant proper Crackle exhibits four turning points, corresponding to the four real roots of $P_{4}(s)$.

We could still be more restrictive and argue that a reasonable definition of constant proper acceleration should also include the vanishing of the Jerk. In such case the observer with constant proper acceleration would be characterized by $\kappa_{2}=0$ and $\kappa_{1}=$ constant. As a matter of fact, the example of circular orbits in Schwarzschild spcetime (see Appendix C) ) favors the adoption of such restrictive proposal for the definition of the constantly accelerated observer.

Summing up, we may contemplate three possible characterizations of the constantly accelerated observer in curved spacetime, from the least to the most restrictive.

a) All curvature invariants are constant.

b) $\kappa_{3}=0$ and $\kappa_{1}, \kappa_{2}$ are constant with $\kappa_{2}<\kappa_{1}$ (our definition in section VIA).

c) $\kappa_{2}=0$ and $\kappa_{1}$ is constant ( $\Leftrightarrow$ vanishing Jerk).

Whereas the second case b) is supported by our procedure in section II, we think that the case c) may be the most physically compelling. To implement this case in our formalism we should simply require the pure boosts that send the observer to rest at different times, to commute among themselves.

We have studied the generalization of this concept of the accelerated observer (that is, with constant proper 1-acceleration) to higher order time derivatives in Minkowski spacetime. All these new concepts allow for immediate extension to curved spacetime. The notions of vanishing $n$-acceleration and of constant proper $n$-acceleration are discussed in detail for the cases of Jerk and Snap. We prove that the vanishing of the Jerk implies that the proper acceleration is constant and in addition that the trajectory lies in a plane $(2 \mathrm{~d})$ in Minkowski spacetime. In contrast, we also show that the vanishing of the Snap does not 
imply that the proper Jerk is constant, except if we restrict the motion to a plane $(2 \mathrm{~d})$.

Although we have proved it only for $n=1, n=2$, we think it very likely that with higher order objects the notions of vanishing $(n+1)$-acceleration and of constant proper $n$ acceleration are equivalent only when the trajectory in Minkowski spacetime lies in a plane (or when $\kappa_{2}=0$ if we consider also curved spacetime). In general, it is not difficult to see that the notion of vanishing $n$-acceleration has a specific characterization in the framework of the Frenet Serret formalism. We believe that the notion of constant proper $n$-acceleration, has also its characterization in such formalism, although we have not proved it beyond the case of the constant proper acceleration. This would mean that all our results would be exportable to curved spacetime. We leave these issues for future work.

In Minkowski spacetime, when we specialize our results to trajectories in a plane, the characterization of vanishing $(n+1)$-acceleration together with constant proper $n$-acceleration is very simple by making use of the Frenet-Serret formalism. It all boils down to require that the second curvature invariant $\kappa_{2}$ vanishes and that the first curvature invariant $\kappa_{1}$ be (the absolute value of) a polynomial of degree $(n-1)$ of the proper time parameter, This result can be exported to generally covariant metric theories with a connection satisfying the metricity condition.

Concerning motion in a plane, we may compare our results with those in Galilean mechanics. In Galilean mechanics with one space dimension, the velocity of an observer with constant proper $n$-acceleration is a $n$-degree polynomial $P_{n}(t)$ of the physical time $t$. In Minkowski $2 \mathrm{~d}$ spacetime the velocity of an observer with constant proper $n$-acceleration is $v(t)=\tanh \left(P_{n}(s(t))\right)$ where $P_{n}$ is an $n$-degree polynomial and $s(t)$-proper time as function

of coordinate time- is obtained after solving the differential equation $\frac{d t}{d s}=\cosh \left(P_{n}(s)\right)$. Thus moving form Galilean to Minkowskian observers amounts to encapsulate the Galilean results within specific hyperbolic trigonometric functions that set up the bound of the speed of light.

\section{ACKNOWLEDGEMENTS}

JMP thanks Joaquím Gomis and Carles Batlle for useful conversations. He also acknowledges financial support from projects 2017-SGR-929, MINECO grant FPA2016-76005-C2-1P and the MDM-2014-0369 of ICCUB (Unidad de Excelencia 'María de Maeztu'). 


\section{Appendix A: Uniform circular motion}

Consider in a $3 \mathrm{~d}$ Minkowski spacetime the trajectory $(r>0$ and $\omega>0$ are constant)

$$
X(t(s))=\left(\begin{array}{c}
t(s) \\
r \sin (\omega t(s)) \\
-r \cos (\omega t(s))
\end{array}\right)
$$

Its velocity with respect to proper time $s$ is

$$
V(s)=\dot{t}(s)\left(\begin{array}{c}
1 \\
r \omega \cos (\omega t(s)) \\
r \omega \sin (\omega t(s))
\end{array}\right)
$$

and the condition $(V)^{2}=-1$ determines $\dot{t}(s)=\frac{1}{\sqrt{1-r^{2} \omega^{2}}} \equiv \gamma$. Note that $r, \omega$ are required to satisfy $r \omega<1$.

The curvature invariant $\kappa_{3}$ vanishes -the motion is $3 \mathrm{~d}$ - and we obtain $\kappa_{1}=\frac{r \omega^{2}}{1-r^{2} \omega^{2}}, \kappa_{2}=$ $\frac{\omega}{1-r^{2} \omega^{2}}$. Thus $\kappa_{1}=r \omega \kappa_{2}<\kappa_{2}$. Adopting the procedure in section VI A we can easily recover from the constancy of $\kappa_{1}\left(<\kappa_{2}\right)$ and $\kappa_{2}$ the solution A.1.

One can systematically compute the $n$-accelerations. Let us define the unitary, spacelike vectors $T$ and $N$ (the spatial component of $T$ is tangent to the spatial component of $V$ and the spatial component of $N$ is normal to the spatial component of $V$ ),

$$
T=\gamma\left(\begin{array}{c}
r \omega \\
\cos (\omega t(s)) \\
\sin (\omega t(s))
\end{array}\right), \quad N=\left(\begin{array}{c}
0 \\
-\sin (\omega t(s)) \\
\cos (\omega t(s))
\end{array}\right)
$$

So we have $T^{2}=N^{2}=1,(T, N)=(T, V)=(N, V)=0$. It is then a matter of straightforward computation to see that the $n$-accelerations $A_{n}$ can be written as

$$
\begin{gathered}
A_{2 k+1}=(-)^{k} r \omega\left(\gamma^{2} \omega\right)^{2 k+1} N, k=0,1,2 \ldots \\
A_{2 k}=(-)^{k} r \omega\left(\gamma^{2} \omega\right)^{2 k} T, k=1,2,3 \ldots
\end{gathered}
$$

Thus in this simple case of uniform circular motion all the $n$-accelerations are non-vanishing, though the norm of each one is constant.

The proper $n$-acceleration is obtained by applying to $A_{n}$ the pure boost (IV.12). Noticing that $B N=N$ and $B T=\gamma(T-r \omega V)$ we observe that all the proper $n$-accelerations $A_{n}^{[p]}$ exhibit a dependence of periodic type on proper time. 


\section{Appendix B: Constantly accelerated observers in an expanding de Sitter universe}

We consider the de Sitter metric with flat equal-time slices,

$$
d s^{2}=-d t^{2}+\left(\mathrm{e}^{\sqrt{\frac{\Lambda}{3}}} t\right)^{2}\left(d x^{2}+d y^{2}+d z^{2}\right)
$$

where $\Lambda$ is the cosmological constant, $\Lambda>0$. With this parametrization, the comoving observers $(\vec{x}=$ constant $)$ are geodesics.

We will show that the timelike trajectory

$$
x(t)=\left(x_{0}-u\right)+u \mathrm{e}^{-\sqrt{\frac{\Lambda}{3}} t}, y=y_{0}, z=z_{0},
$$

(the constant parameter $u$ will be restricted below) satisfying $x(0)=x_{0}$, exhibits vanishing Jerk, which implies constant proper acceleration.

In fact, the velocity with respect to proper time $\left((V)^{2}=V^{\mu} g_{\mu \nu} V^{\nu}=-1\right)$ is

$$
V=\frac{1}{\sqrt{1-\frac{u^{2} \Lambda}{3}}}\left(\begin{array}{c}
1 \\
-u \sqrt{\frac{\Lambda}{3}} \mathrm{e}^{-\sqrt{\frac{\Lambda}{3}} t} \\
0 \\
0
\end{array}\right)
$$

Note that we need $|u|<\sqrt{\frac{3}{\Lambda}}$, otherwise the trajectory becomes lightlike (for $|u|=\sqrt{\frac{3}{\Lambda}}$ ) or spacelike (for $|u|>\sqrt{\frac{3}{\Lambda}}$ ).

Note that the coordinate time is proportional to the proper time, $\frac{d t}{d s}=\frac{1}{\sqrt{1-\frac{u^{2} \Lambda}{3}}}$. The acceleration becomes

$$
A^{\mu}=\frac{d}{d s} V^{\mu}+V^{\rho} \Gamma_{\rho \nu}^{\mu} V^{\nu}=\frac{1}{\sqrt{1-\frac{u^{2} \Lambda}{3}}} \frac{d}{d t} V^{\mu}+V^{\rho} \Gamma_{\rho \nu}^{\mu} V^{\nu}=\left(\begin{array}{c}
\frac{u^{2} \Lambda^{3 / 2}}{\sqrt{3}\left(3-u^{2} \Lambda\right)} \\
-\frac{u \Lambda}{3-u^{2} \Lambda} \mathrm{e}^{-\sqrt{\frac{\Lambda}{3}} t} \\
0 \\
0
\end{array}\right),
$$

which satisfies

$$
(A)^{2}=A^{\mu} g_{\mu \nu} A^{\nu}=\frac{u^{2} \Lambda^{2}}{9-3 u^{2} \Lambda} .
$$

Note that for $u=0$ the motion is geodesic. Finally, we check that the Jerk vanishes,

$$
\Sigma^{\mu}=\frac{d}{d s} A^{\mu}+V^{\rho} \Gamma_{\rho \nu}^{\mu} A^{\nu}-(A)^{2} V^{\mu}=0 .
$$

One can compute also curvature invariants. We obtain $\kappa_{1}=\frac{u \Lambda}{\sqrt{9-3 u^{2} \Lambda}}, \kappa_{2}=0$. We may notice that, in view of (VI.4), which is valid for our curved spacetime, the constancy of $\kappa_{1}$ and the vanishing of $\kappa_{2}$ already guarantee the vanishing of the Jerk. 


\section{Horizon for the accelerated observer}

Now we compare the trajectory of our accelerated observer with that of light. In the $x$ direction the two lightlike trajectories with initial condition $\bar{x}(0)=\bar{x}_{0}$ are

$$
\bar{x}(t)=\left(\bar{x}_{0} \mp \sqrt{\frac{3}{\Lambda}}\right) \pm \sqrt{\frac{3}{\Lambda}} \mathrm{e}^{-\sqrt{\frac{\Lambda}{3}} t}, \bar{y}=y_{0}, \bar{z}=z_{0},
$$

so that $\bar{x}(0)=\bar{x}_{0}$.

Let us find conditions on $x_{0}$ and $\bar{x}_{0}$ to guarantee that at some moment in the future or in the past both trajectories intersect. The geodesic line between the two trajectories taken at time $t_{0}$ is, for the $x$ coordinate $(\lambda \in[0,1])$

$$
\hat{x}(\lambda)=\lambda x\left(t_{0}\right)+(1-\lambda) \bar{x}\left(t_{0}\right)
$$

and $\hat{y}(\lambda)=0, \hat{z}(\lambda)=0$. The distance $d$ at $t_{0}$ between the two trajectories at this time $t_{0}$ is

$$
d\left(\bar{x}\left(t_{0}\right), x\left(t_{0}\right)\right)=\int_{0}^{1} d \lambda \sqrt{\frac{d \hat{x}}{d \lambda} g_{x x} \frac{d \hat{x}}{d \lambda}}=\left|\left(\bar{x}\left(t_{0}\right)-x\left(t_{0}\right)\right) \mathrm{e}^{\sqrt{\frac{\Lambda}{3}} t_{0}}\right| .
$$

For this distance to vanish at some finite time $t_{0}$ we need

$$
0=\left|\left(\bar{x}\left(t_{0}\right)-x\left(t_{0}\right)\right) \mathrm{e}^{\sqrt{\frac{\Lambda}{3}} t_{0}}\right|=\left|\left(\bar{x}_{0}-x_{0}+\left(u \mp \sqrt{\frac{3}{\Lambda}}\right)\right) \mathrm{e}^{\sqrt{\frac{\Lambda}{3}} t_{0}}-\left(u \mp \sqrt{\frac{3}{\Lambda}}\right)\right|
$$

and this equation has solution for $t_{0}$ if and only if

$$
\frac{\bar{x}_{0}-x_{0}+\left(u \mp \sqrt{\frac{3}{\Lambda}}\right)}{\left(u \mp \sqrt{\frac{3}{\Lambda}}\right)}>0,
$$

which is equivalent to

$$
\left|\bar{x}_{0}-\left(x_{0}-u\right)\right|<\sqrt{\frac{3}{\Lambda}} .
$$

This is the condition for the accelerated observer and lightlike trajectories to intersect somewehere. The non-intersection condition sets up a horizon -here at time $t=0$ - located at $\bar{x}_{0}=x_{H}(0)$

$$
x_{H}(0)=\left(x_{0}-u\right) \pm \sqrt{\frac{3}{\Lambda}} .
$$

This horizon moves at the speed of light,

$$
x_{H}(t)=\left(x_{0}-u\right) \pm \sqrt{\frac{3}{\Lambda}} \mathrm{e}^{-\sqrt{\frac{\Lambda}{3}} t} .
$$

The picture given by (B.5) is incomplete and can be misleading since it seems that there are two horizons. In view of B.2 and B.5 it is convenient to adopt spherical coordinates 
for the equal-time 3 -surfaces centered in $x=x_{0}-u, y=y_{0}, z=z_{0}$. With $r$ the radial coordinate, the trajectory of the accelerated observer is then

$$
r(t)=u \mathrm{e}^{-\sqrt{\frac{\pi}{3}} t},
$$

with the angular variables remaining fixed. Now the horizon appears in the equal-time surfaces as a sphere around $x_{0}-u, y=y_{0}, z=z_{0}$ with coordinate radius

$$
r_{H}(t)=\sqrt{\frac{3}{\Lambda}} \mathrm{e}^{-\sqrt{\frac{\Lambda}{3}} t} .
$$

moving at the speed of light. Notice that this is nothing but the future event horizon [10], also known as the cosmological horizon of de Sitter.

Note that for $t \rightarrow \infty$ both $r(t)$ and $r_{H}(t)$ tend to $r=0$, but this is an effect of the coordinatization. As a matter of fact all equal-time distances between the center $r=0$, the trajectory of the accelerated observer, and the horizon, remain constant. For instance the distance between the trajectory and the horizon is, for the same values of the angular variables,

$$
D\left(r(t), r_{H}(t)\right)=\left|\left(r(t)-r_{H}(t)\right) \mathrm{e}^{\sqrt{\frac{\Lambda}{3}} t}\right|=\sqrt{\frac{3}{\Lambda}}-u .
$$

whereas the distance of the accelerated observer to the center is $u$ and the length of the radius of the spherical horizon is $\sqrt{\frac{3}{\Lambda}}$.

\section{Comoving observers of different kind}

In fact one can use (B.6) to define the standard static coordinates for de Sitter. We know that the comoving observers for the metric (B.1) are geodesics, and we also know that the radial motion that keeps $r \mathrm{e}^{\sqrt{\frac{1}{3}} t}$ constant is that of a constantly accelerated observer. If we define the new radial coordinate $u=r \mathrm{e}^{\sqrt{\frac{1}{3}} t}$ then this accelerated observer will sit at constant $u$. The metric becomes

$$
\begin{array}{r}
d s^{2}=-d t^{2}+\mathrm{e}^{\sqrt{\frac{\Lambda}{3}} t}\left(d r^{2}+r^{2}\left(d \theta^{2}+(\sin \theta)^{2} d \varphi^{2}\right)\right) \rightarrow \\
-\left(1-\frac{\Lambda}{3} u^{2}\right) d t^{2}+d u^{2}-2 u \sqrt{\frac{\Lambda}{3}} d t d u+u^{2}\left(d \theta^{2}+(\sin \theta)^{2} d \varphi^{2}\right) .
\end{array}
$$

Next, to get rid of the non-diagonal term, we solve a differential equation and obtain the change of variables $T=t-\frac{1}{2} \sqrt{\frac{3}{\Lambda}} \log \left(1-\frac{\Lambda}{3} u^{2}\right)$. We get

$$
d s^{2}=-\left(1-\frac{\Lambda}{3} u^{2}\right) d T^{2}+\frac{1}{1-\frac{\Lambda}{3} u^{2}} d u^{2}+u^{2}\left(d \theta^{2}+(\sin \theta)^{2} d \varphi^{2}\right) .
$$

So, in de Sitter spacetime, whereas the comoving observers in the coordinatization of (B.1) are geodesics, the comoving observers in (B.8) are constantly accelerated observers. 
A similar construction can be performed for Anti de Sitter spacetime. The comoving observers in the coordinates of the metric $(\overline{B .8})$, now with $\Lambda<0$, exhibit constant proper acceleration. Looking for the radial geodesics in this form of the metric we can move to a coordinatization in which such geodesics are the trajectories of the comoving observers. Unsurprisingly, one obtains the "cosmological" form of AdS metric $\left(a=\sqrt{-\frac{\Lambda}{3}}\right)$,

$$
d s^{2}=-d t^{2}+(\cos (a t))^{2}\left(\frac{1}{1+a^{2} r^{2}} d r^{2}+r^{2}\left(d \theta^{2}+(\sin \theta)^{2} d \varphi^{2}\right)\right) .
$$

\section{Appendix C: Circular orbits in Schwarzschild spcetime}

With coordinates $(t, r, \theta, \varphi)$, we consider Schwarzschild metric

$$
\left.d s^{2}=-\left(1-\frac{2 M}{r}\right) d t^{2}+\frac{1}{\left(1-\frac{2 M}{r}\right)} d r^{2}+r^{2}\left(d \theta^{2}+(\sin \theta)^{2} d \varphi^{2}\right)\right),
$$

and circular uniform timelike trajectories $\left(t, r_{0}, \frac{\pi}{2}, \omega t\right)$ outside the event horizon, $r_{0}>2 M$. In terms of proper time $s$, the velocity vector is $V=\dot{t}(s)(1,0,0, \omega)$ and the requirement $V^{2}=-1$ yields $\dot{t}=\left(1-\frac{2 M}{r_{0}}-r_{0}^{2} \omega^{2}\right)^{-\frac{1}{2}}$ which sets the bound for $\omega$,

$$
\omega^{2}<\frac{r_{0}-2 M}{r_{0}^{3}},
$$

to keep the trajectory timelike. Using the definitions in section VI, extended through section IIE to curved spacetime, it is easy to compute the curvature scalars. We get

$$
\kappa_{1}=\frac{\sqrt{r_{0}-2 M\left|M-r_{0}^{3} \omega^{2}\right|}}{r_{0}^{\frac{3}{2}}\left(r_{0}-2 M-r_{0}^{3} \omega^{2}\right)}, \quad \kappa_{2}=\frac{\omega\left|r_{0}-3 M\right|}{\left(r_{0}-2 M-r_{0}^{3} \omega^{2}\right)}, \quad \kappa_{3}=0 .
$$

The condition $\kappa_{1}=0$, that is, $r_{0}^{3} \omega^{2}=M$, identifies geodesic motion for our circular orbits. In this case, since $\omega$ already satifies (C.2) we end up with the well known condition $r_{0}>3 M$ for the existence of timelike geodesics in circular motion (the circular geodesic becomes lightlike for $\left.r_{0}=3 \mathrm{M}\right)$. Now we have the aditional information that the second curvature invariant vanishes for circular orbits at $r_{0}=3 \mathrm{M}$. For these orbits at $r_{0}=3 \mathrm{M}$ to be timelike it suffices to require $\omega^{2}<\frac{M}{r_{0}^{3}}$. Thus these circular orbits, with constant $\kappa_{1}$ and vanishing $\kappa_{2}$ correspond to our most restrictive version of constantly accelerated observers.

Let us turn to the less restrictive version considered in sections IIC, VIA, so that we require $\kappa_{1}$ and $\kappa_{2}$ to be constant but with $\kappa_{2}<\kappa_{1}$. Let us compute the quantity (with $\left.X:=r_{0}^{3} \omega^{2}<r_{0}-2 M\right)$

$$
Q=\left(\frac{\kappa_{2}}{\kappa_{1}}\right)^{2}=\frac{X\left(r_{0}-3 M\right)^{2}}{\left(r_{0}-2 M\right)(M-X)^{2}},
$$


and ask for values of $X$, compatible with $X<r_{0}-2 M$, such that $Q<1$. We find
a) for $r_{0}>3 M$, any $X$ chosen as $X<\frac{M^{2}}{r_{0}-2 M}$,
b) for $2 M<r_{0} \leq 3 M$, any $X$ chosen as $X<r_{0}-2 M$.

Note that in both cases $X<M$ (recall that $X=M$ is geodesic motion, which in fact is impossible for time like trajectories in the case $\mathbf{b})$ ). The $\mathbf{b}$ ) case is quite understanable: $\omega$ is allowed to have any value compatible with the speed of light bound $X<r_{0}-2 M$. Instead, the requirement $X<\frac{M^{2}}{r_{0}-2 M}$ for the case a) could make us think that something special happens at the "critical" value $X=\frac{M^{2}}{r_{0}-2 M}$; we do not see anything special at this value for $X$, which makes $\kappa_{2}=\kappa_{1}=\frac{M \sqrt{r_{0}-2 M}}{r_{0}^{3 / 2}\left(r_{0}-M\right)}$. Thus if this "critical" value has no meaning other than that, it would seem more natural to adopt the most restrictive version of the notion of the constantly accelerated observer, that is, the vanishing of $\kappa_{2}$ and constancy of $\kappa_{1}$. This singles out, in the case of circular orbits, those at $r_{0}=3 M$.

[1] W. G. Unruh, "Notes on black hole evaporation," Phys. Rev. D 14 (1976) 870. doi:10.1103/PhysRevD.14.870

[2] L. C. B. Crispino, A. Higuchi and G. E. A. Matsas, "The Unruh effect and its applications," Rev. Mod. Phys. 80 (2008) 787 doi:10.1103/RevModPhys.80.787 [arXiv:0710.5373 [gr-qc]].

[3] G. Cozzella, A. G. S. Landulfo, G. E. A. Matsas and D. A. T. Vanzella, "A quest for a "direct" observation of the Unruh effect with classical electrodynamics: In honor of Atsushi Higuchi 60th anniversary," Int. J. Mod. Phys. D 27 (2018) no.11, 1843008 doi:10.1142/S0218271818430083 [arXiv:1803.05059 [gr-qc]].

[4] M. Dunajski and G. Gibbons, "Cosmic Jerk, Snap and Beyond," Class. Quant. Grav. 25, 235012 (2008) doi:10.1088/0264-9381/25/23/235012 [arXiv:0807.0207 [gr-qc]].

[5] J. G. Russo and P. K. Townsend, "Relativistic Kinematics and Stationary Motions," J. Phys. A 42 (2009) 445402 doi:10.1088/1751-8113/42/44/445402 [arXiv:0902.4243 [hep-th]].

[6] Y. Friedman and T. Scarr, "Uniform Acceleration in General Relativity," Gen. Rel. Grav. 47 (2015) 121 doi:10.1007/s10714-015-1966-5 [arXiv:1602.03067 [gr-qc]].

[7] F. W. Hehl, J. D. McCrea, E. W. Mielke and Y. Ne'eman, "Metric affine gauge theory of gravity: Field equations, Noether identities, world spinors, and breaking of dilation invariance," Phys. Rept. 258, 1 (1995) doi:10.1016/0370-1573(94)00111-F [gr-qc/9402012].

[8] J. R. Letaw, "Vacuum Excitation of Noninertial Detectors on Stationary World Lines," Phys. Rev. D 23 (1981) 1709. doi:10.1103/PhysRevD.23.1709

[9] E. Ramos and J. Roca, "Extended gauge invariance in geometrical particle models and the geometry of W symmetry," Nucl. Phys. B 452 (1995) 705 doi:10.1016/0550-3213(95)00329-Q 
[hep-th/9504071].

[10] S. W. Hawking and G. F. R. Ellis, "The Large Scale Structure of Space-Time," doi:10.1017/CBO9780511524646

[11] D. de la Fuente and, A. Romero, "Uniformly accelerated motion in General Relativity: completeness of inextensibe trajectories," Gen. Relativ. Gravit. 47 (2015) 33, doi:10.1007/s10714015-1879-3 